\title{
Development of an adaptive template for fast detection of lithographic patterns of light-emitting diode chips
}

\section{Wei-Han Weng}

National Taiwan University of Science and Technology

Chen-Yang Tsai

MPI Corporation

Cheng-Yu Hung

National Taiwan University of Science and Technology

Chung-Feng Jeffrey Kuo ( $\boldsymbol{D}$ jeffreykuo@mail.ntust.edu.tw )

National Taiwan University of Science and Technology

\section{Research Article}

Keywords: Light-emitting diode, Scale-invariant feature, Grayscale entropy, Correlation coefficient, Adaptive template, Receiver operating characteristic curve

Posted Date: February 24th, 2021

DOI: https://doi.org/10.21203/rs.3.rs-225589/v1

License: (c) (i) This work is licensed under a Creative Commons Attribution 4.0 International License. Read Full License 


\title{
Development of an adaptive template for fast detection of lithographic patterns of light-emitting diode chips
}

\author{
Wei-Han Weng ${ }^{1}$, Chen-Yang Tsai ${ }^{2}$, Cheng-Yu Hung ${ }^{1}$, Chung-Feng Jeffrey Kuo ${ }^{*}, 1$ \\ ${ }^{1}$ Department of Materials Science \& Engineering, National Taiwan University of \\ Science and Technology, Taipei 106, Taiwan \\ ${ }^{2}$ Photonics Automation Division, MPI Corporation, Chu-Pei City, Hsinchu County 302, \\ Taiwan
}

*Corresponding author: Email: jeffreykuo@mail.ntust.edu.tw

\begin{abstract}
With the expansion in light-emitting diode (LED) lighting market and technology, control of product quality has become the focus of LED development. To achieve high online production capacity, automated quality detection with object image has been employed for comparison using mostly the standard templates. However, the resulting poor fitting causes misjudgment of the detection system. This study proposes an adaptive template method to improve the system fitting, reduce the system misjudgment, and enhance the detection efficiency. The severely damaged LED chips were screened out based on their grayscale entropy indices and related coefficient indices, which enhanced the reliability of the adaptive template system and accelerated the overall system detection process. To overcome the displacement and scale changes of the lithographic patterns, the scale-invariant feature transform (SIFT) and Harris-Laplace methods were used for comparison. The scale-invariant feature transform (SIFT) and Harris-Laplace methods were used to search and compare the feature points of the chip patterns, with the aim to overcome the displacement and scale changes of the lithographic patterns and establish the adaptive template in real conditions. The fast correlation coefficient comparison method was compared with the adaptive template comparison method proposed in this study. The results showed that the detection accuracy of the new method was $98.36 \%$, which is $15.79 \%$ more accurate than the fast correlation coefficient comparison method. In terms of time performance, the method proposed in this study took $0.08 \mathrm{~s}$ less to complete the partition defect template. Moreover, the average detection time per chip was reduced by another $1.4 \mathrm{~s}$, which improved the efficiency by $30.43 \%$. The adaptive template proposed in this study exclusively establish itself based on different lithographic patterns, thus improving the detection efficiency and accuracy of the LED industry, and raising its market competitiveness in the industry.
\end{abstract}


Keywords: Light-emitting diode, Scale-invariant feature, Grayscale entropy, Correlation coefficient, Adaptive template, Receiver operating characteristic curve

\section{Introduction}

In recent years, energy demand has increased across the world, leading to rising prices. Energy-saving and high-efficiency lighting has gradually become the focus of energy development [1]. The light-emitting diode (LED) has the advantages of low energy consumption, high brightness and long service life [2], which are a new development focus in lighting systems [3]. To form lithographic patterns on LED chips, as shown in Figure 1(a), lithographic optical and chemical etching procedures are required, with a variety of light masks [4] and optical path projection systems [5]. However, in the lithographic optical process, the poor location of the lithographic masks and the poor quality of wafer result in the displacement and scale changes of the different layers of light mask patterns. The lithographic optical system is shown in Fig. 1(a). In Fig. 1(b), there is a significant displacement of the two chips on the lithographic patterns. When the standard template is used for detection, there will be dismatching as leakage or overkill during detection due to poor fitting. However, once the displacement of the object has been overcome, the templated matching could be considered as a fast detection method [6]. 


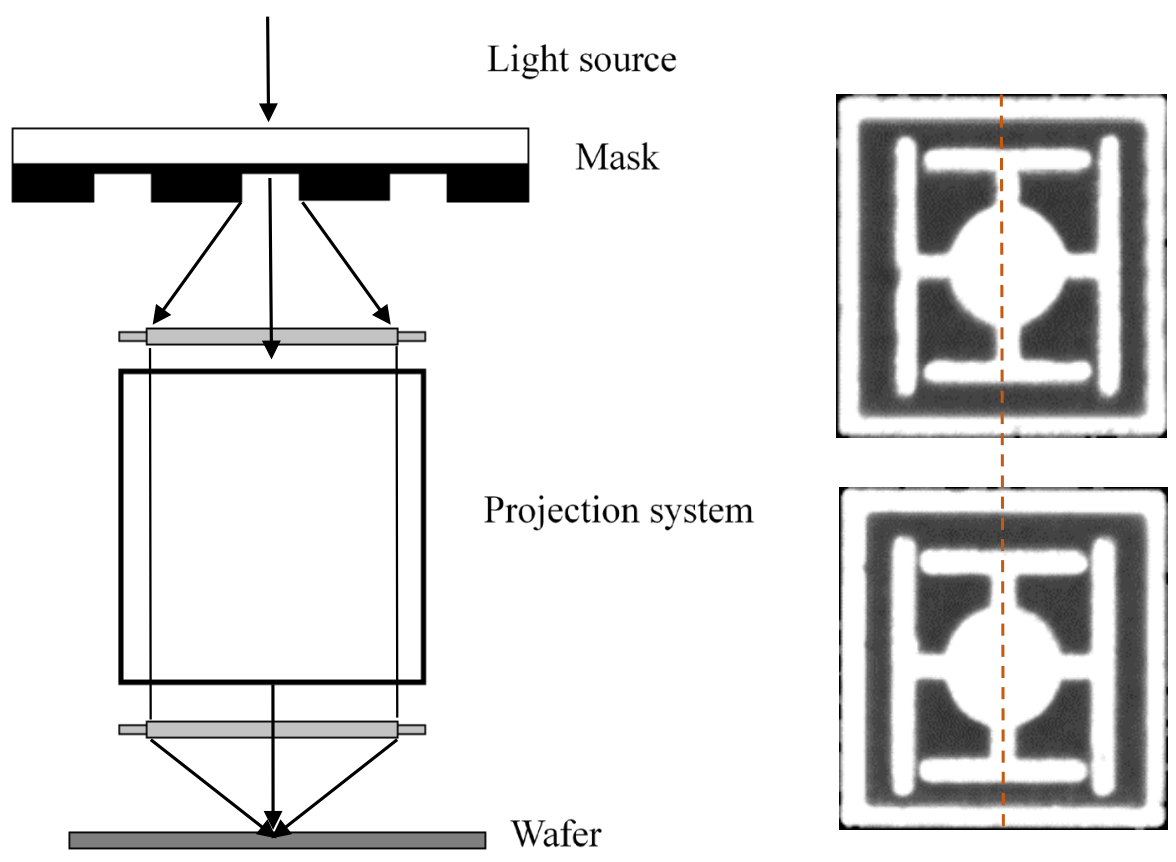

(a) Lithographic optical system

(b) Displacement of lithographic patterns

Fig. 1. Lithographic optical system and displacement of lithographic patterns

For quality inspection, automatic inspection by machine vision method is now used, since considerable personnel resources may generate corresponding misjudgment due to human fatigue [7]. The measurement of the lithographic mask variation in each layer of LED chips is mainly divided into two steps: segmentation of image blocks and location of feature points. The lithographic patterns of LED chips include the finger electrode area, pad electrode area, light-emitting area, and mesa area, as shown in Fig. 2. In practical application, three problems arise during object detection: (1) variant scale of object, (2) deformations of intra-class objects, (3) cluttered edges between the background and object. These problems can lead to confusion during object location and detection [8]. For the best segmentation index to extract the lithographic patterns in each layer, related research on location of feature points is divided into the areabased method $[9,10,11]$ and the feature-based method $[12,13,14]$.

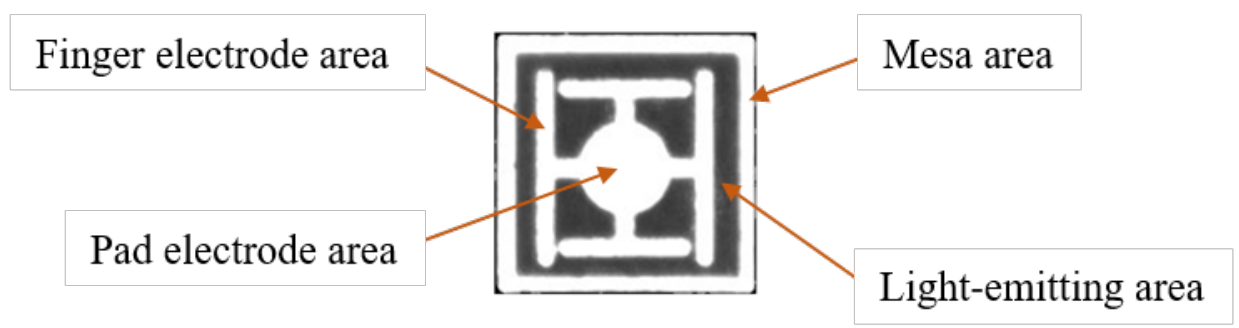


Fig. 2. Chip layers

The area-based method refers to finding the correlation coefficient of a specific area between two images. The fast normalized cross correlation (FNCC) is the most famous method for finding this relationship [15]. It is widely used for similar inspection and dectetion [16]. Kuo et al. [17] proposed a robust template matching technique to improve the time and accuracy for printed circuit boards (PCB). The results proved that the template matching technique not only has sub-pixel level high accuracy and short computing time but also robust rotation change and scale change interference. Stefano et al. [18] proposed an area-based stereo algorithm, referred to as a single matching phase (SMP), which is suitable for real-time applications. The algorithm is based on core matching and checks the validity of a match directly at the comparison stage without reverse matching. We know, from the literature, that once the correlation coefficient has been normalized, it becomes more stable and suitable for rapid template comparison. Wang et al.[19] presented the camera calibration algorithm of the LED chip visual positioning system to improve the chip positioning accuracy. Through error analysis of the visual positioning system, the system errors of each part of the system were obtained, and the relationship between the chip positioning error and the chip position distribution in the image was found. Then, based on the results of error analysis and the characteristics of the chip positioning process, an improved calibration algorithm was proposed to improve the chip positioning accuracy Perng et al. [20] demonstrated a machine vision system combined the inspection area (IR) method automatically generated to inspect two types of LED surface mount devices (SMD). The proposed automatic inspection method could acahieve 95\% accuracy. The detect defects included missing components, wrong orientation, reverse polarity, mouse bites, missing gold wires and surface stains.

For the feature-based method, the main instructions for finding the key points are: 1) the dominant gradient orientation of the local interest region around the feature points must be estimated from the local appearance and geometry; 2) the direction of the line linking two nearby feature points must be consistent with the local geometry [21][22]. Harris et al. [23] proposed an algorithm for detecting corner points and edge points in separate studies of research [24][25]. They used the quadratic function eigenvalue formed by Taylor expansion and similarity comparison in the detection template to determine whether the corresponding points in the template were corner points, and then screened out the feature points based on the corner points. Smith and Brady [26] proposed a set of corner point detection methods to calculate the pixel changes and extract the corner points with a detection template, using the path movement mode [27], 
which is also called the Susan corner point operation. In the Harris and Susan feature point detection, the corner point operator only has displacement-, grayscale- and rotation-invariant features, but does not have a scale-invariant feature. To apply the feature point comparison to the objects with scale changes, as there are scale changes between sample layers in this study, the scale-invariant search method is adopted. Zhang et al. [28] used blob analyzation-based template matching algorithm for LED chip localization to solve the elimination of polycrystalline and fragmented LED chips. The image segmentation method was used to obtain spots, exclude abnormal spots and predict the pose (position and direction) of potential objects. Precise positioning of LED chips based on gradient direction characteristics. Yan et al. [29] detected typical surface defects of aluminum alloy welds, the structured laser was responsible for obtaining 3D depth images of the bead surface. The multi-angle illumination was ued to capture grayscale images. According to the different features shown in the 3D depth map and the 2D grayscale map, extracting the weld boundary were presented. Lowe [30] applied the scale-invariant feature transform (SIFT) method, which extracted feature points through Gaussian pyramid decomposition and Difference of Gaussians (DoG) [31][32], and then generated feature vectors to search the features. The SIFT method could provide rotation- and scale-invariant features, and achieve robustness against environmental interference. A subsequent study by Se et al. [33] employed the SIFT method to address the combination of location and reality maps. The feature points of multiple maps could be connected through the algorithm to explore the environment. This algorithm is currently used to build reality maps [34]-[36]. The Harris-Laplace algorithm method was developed to extract the scale-invariant feature points [37]-[39]. Through the image pyramid decomposition and DoG calculation, Harris corner point detection can be used to search layered regional feature points. Specifically, the optimal effect is to decompose the four-level scale factors, and the extracted normalized feature points are invariant in terms of scale, rotation, and displacement [40][41]. SIFT and other invariant moments have been more recently cited for comparison, due to their reproducibility. Therefore, in this study, the SIFT and Harris-Laplace methods have been used to explore the scale-invariant moments. Zhang et al. [42] improved the Harris-Laplace method for higher repeatability, using all the points in the image with the largest scale in the scale-space as tracked and grouped. The experimental results showed the effectiveness of the inspection, with an average inspection time of $8.78 \mathrm{~ms}$ per die and an average accuracy $92.4 \%$.

The shortcoming of the SIFT and Harris-Laplace methods is that the image information calculation workload is huge during the comparison of feature points of the standard images and comparison images, which greatly slows down the comparison process. Therefore, the comparison method must be improved in practice to meet the 
demand for high production speed. To extract the feature points in this study, SIFT and Harris-Laplace methods were used to search for feature points due to their scale invariance, and to detect the feature points of the chip patterns. Correlation coefficient matching was used to obtain the integrity index of the LED chip layers, and compared to template matching for fast pre-screening. The feature points were quickly searched and accurately located by area-based search. Next, the feature areas were located by the template comparison method. An adaptive template that matched the chip was established based on the location of light masks, in order to overcome the displacement and scale changes between the mask patterns in the different layers, and to achieve the fastest fitting effect.

\section{Methods}

In this study, the SIFT and Harris-Laplace methods were used to search for the feature areas on the LED chip patterns and analyze the LED chip lithographic patterns for adaptive template development. The feature area template comparison method was used to accelerate the location of feature points. Through the lithographic layered information of the located points, an adaptive template was established. To improve the locating accuracy of adaptive template comparison, a fast chip integrity screening algorithm was introduced before the locating process to improve the efficiency of system judgment.

\subsection{Fast chip integrity screening}

Statistical analysis showed that the surface patterns of many of the chips whose images were extracted from the wafer were severely damaged or heavily contaminated, which caused misjudgment of the locating system and increased a lot of meaningless computing workload. Before an adaptive template was established, a fast screening algorithm was introduced to improve its locating feasibility. The process is shown in Fig. 3. 


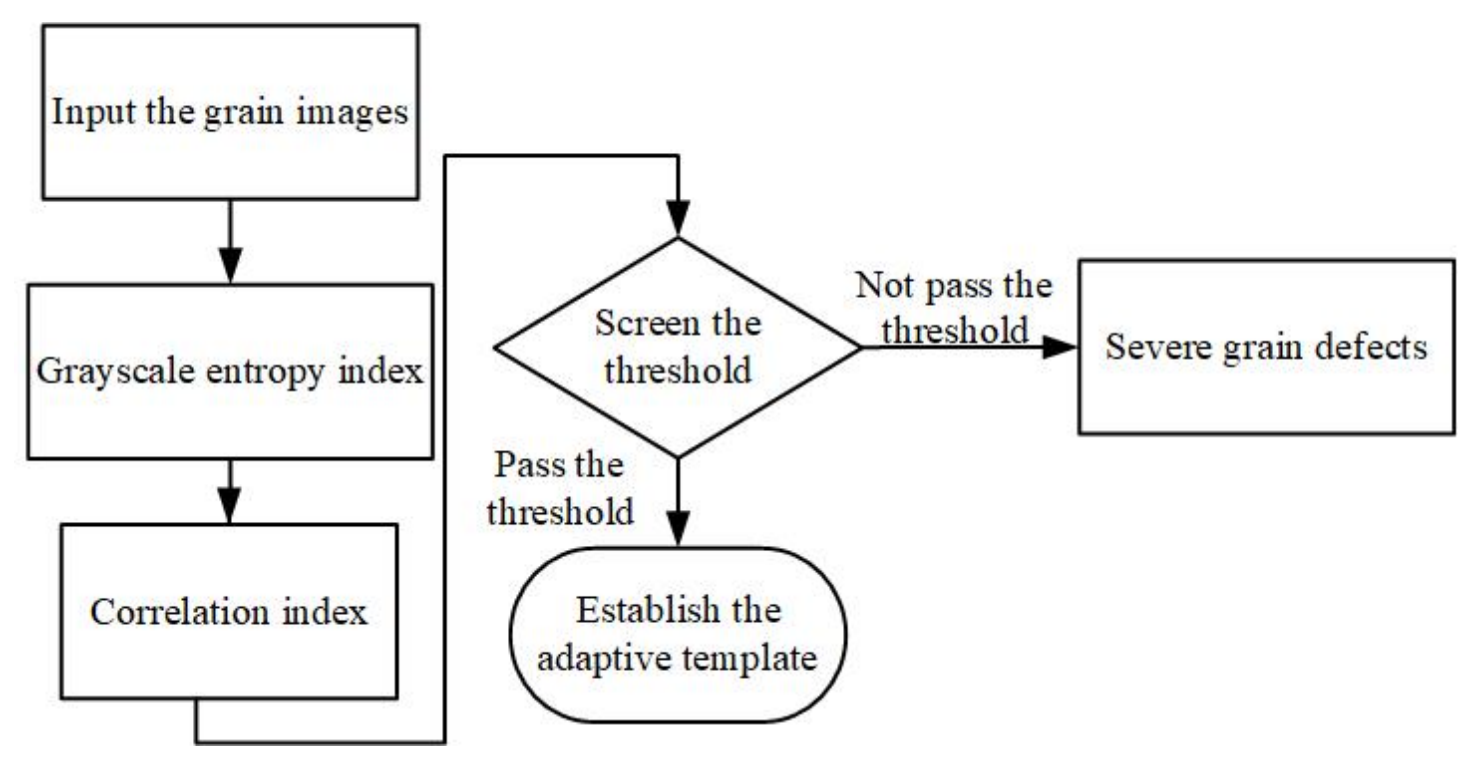

Fig. 3 Fast screening process

\subsection{Grayscale entropy index}

In this study, prior to integrity screening of the chip lithographic patterns, the optimal judgment index of the chip structure was analyzed in advance, and the grayscale distribution and the pattern gradient were compared. There were obvious differences in the grayscales of different areas in the LED chip patterns, as shown in Fig. 4. The chip lithographic patterns extracted by the grayscale distribution were more accurate than those extracted by the pattern gradient.

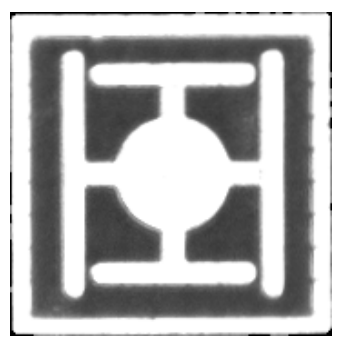

(a) Chip image

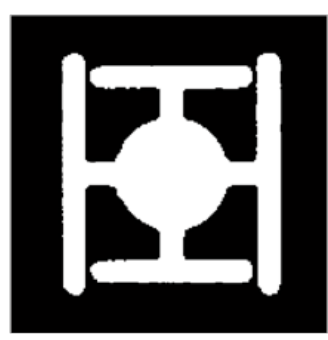

(b) Grayscale index

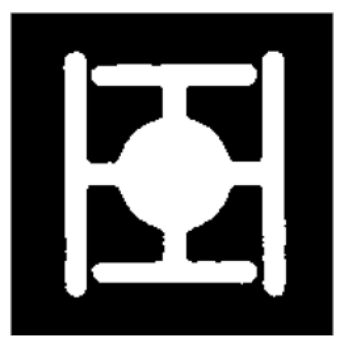

(c) Lithographic pattern

Fig. 4. Grayscale index and pattern index

As shown in Fig. 5, there are obvious peaks in the grayscale histogram of the nondefective samples, while the cumulative peak pixels in the defective chip histogram are lower. Therefore, the defective chips can be identified by histogram analysis of the grayscale distribution indices, which refer to the range marked by the line segment in 
Fig. 5(d).

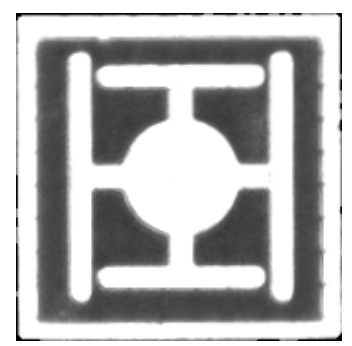

(a) Non-defective

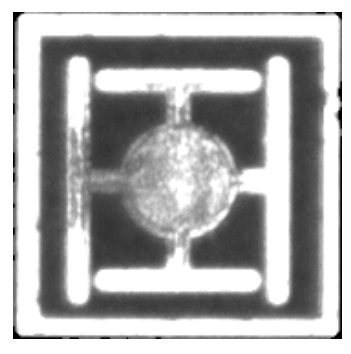

(c) Defective chip

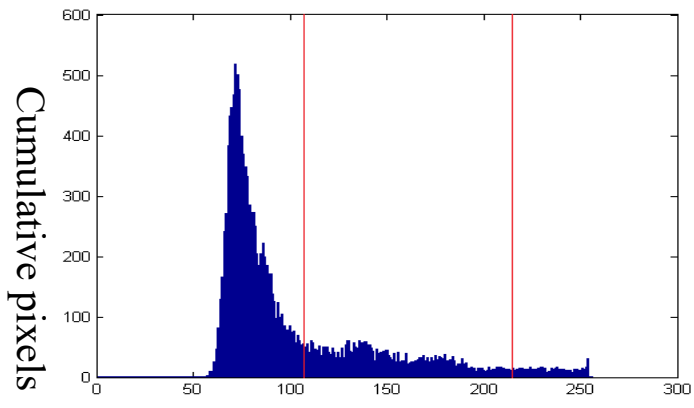

Grayscale range

(b) Grain grayscale

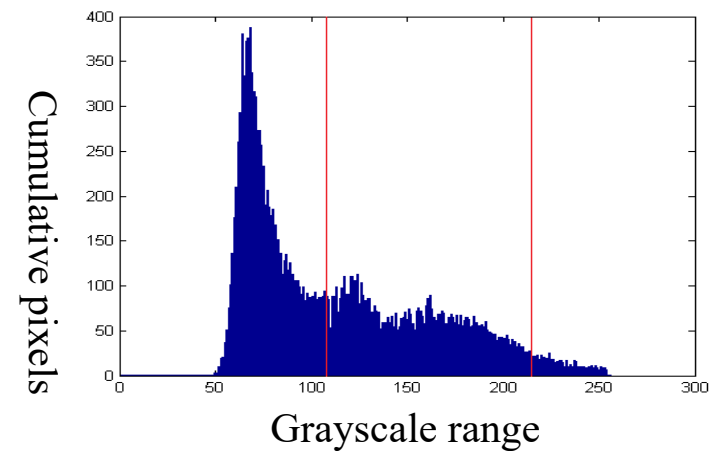

(d) Grain grayscale

Fig. 5. LED chip and grayscale histogram

\subsection{Correlation coefficient index}

As the grayscale index range of the defective chips was close to the grayscale index range of the non-defective chips, it was difficult to fast-screen the severely damaged chips. The correlation coefficient was used as an auxiliary index to screen out the chips with severely damaged lithographic patterns, thus avoiding errors during the subsequent establishment of the adaptive template.

The fast correlation coefficient comparison method could find the correlation between the detected image and the standard template image. For a detected image $f(x, y)$ of size $M \times N$ pixels, when the goal is to search for a standard template image $r(i, j)$ of size $m \times n$ pixels, the process can be achieved using Eqs. (1)-(3).

$$
f(x, y)=\frac{1}{M \times N} \sum_{i=1}^{M} \sum_{j=1}^{N} f(x+i, y+j)
$$




$$
\begin{aligned}
& r(i, j)=\frac{1}{m \times n} \sum_{i=1}^{m} \sum_{j=1}^{n} r(x+i, y+j) \\
& \delta=\frac{\sum_{i=1}^{M} \sum_{i=1}^{N}[(f(x, y)-\bar{f})-(r(x, y)-\bar{r})]}{\mathrm{s}_{\mathrm{f}} \times s_{r}}
\end{aligned}
$$

where $\bar{f}$ is the mean grayscale in the detected image window, $\bar{r}$ is the mean grayscale in the standard image window, and $\mathrm{S}_{\mathrm{f}}$ and $S_{r}$ are the standard deviations of the grayscale in the standard image comparison window.

Due to the displacement and scaling differences between the chip patterns, the blurring effect was introduced in the standard template to relax the parameters of the comparison template and alleviate the poor fitting caused by changes between layers. In the blurring method, which is based on the Gaussian blur function, a convolution integral is evaluated on the standard template image with a Gaussian blur function structure of dimensions $5 \times 5$, as shown in Fig. 6 .

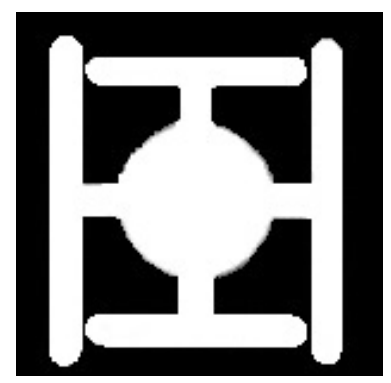

(a) Original template

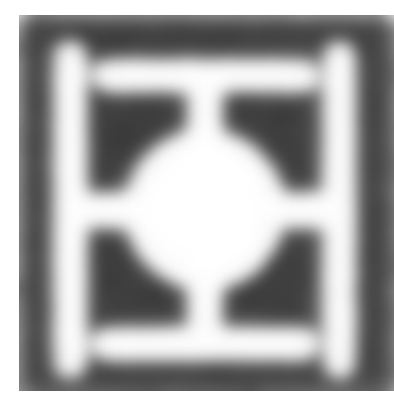

(b) Blurred template

Fig. 6. Template blurring

\subsection{Training of chip feature areas}

The search for feature points must overcome the displacement and scale changes between the layers of the LED chip lithographic patterns. The SIFT and Harris-Laplace methods were adopted to judge and analyze the feature points.

\subsubsection{SIFT feature point search method}

The SIFT algorithm includes scale space generation, feature point extraction, and feature vector statistics. The purpose of feature vector statistics is to calculate the change in the images of the same object using vectors. In the SIFT feature point extraction, the feature points were extracted by the image of the DoG to analyze the 
feature points of a single image. First, the scale smoothing filter function in the image scale space was defined as:

$$
\begin{aligned}
& G(x, y, \sigma)=\frac{1}{2 \pi \sigma^{2}} e^{-\left(x^{2}+y^{2}\right) / 2 \sigma^{2}} \\
& L(x, y, \sigma)=G(x, y, \sigma)^{*} I(x, y)
\end{aligned}
$$

where $G(x, y, \sigma)$ is the two-dimensional Gaussian blur function, $I(x, y)$ is the original image, $L(x, y, \sigma)$ is the scale space, and $\sigma$ is the Gaussian scale factor. The Gaussian blur function was used to evaluate the convolution integral on the original image to obtain the scale space.

Then, the exponential rate of $k$ was introduced into the Gaussian blur function to obtain the blurring effect at different levels and rates. In this study, $k$ was set to $\sqrt{2}$ and imported into different scale spaces. Then, blurred images at adjacent levels were subtracted and convolved with the original images to obtain the Difference of Gaussians space. The schematic diagram is shown in Fig. 7.

$$
\begin{aligned}
D(x, y, \sigma) & =(G(x, y, k \sigma)-G(x, y, \sigma))^{*} I(x, y) \\
& =L(x, y, k \sigma)-L(x, y, \sigma)
\end{aligned}
$$

\section{Level 2}
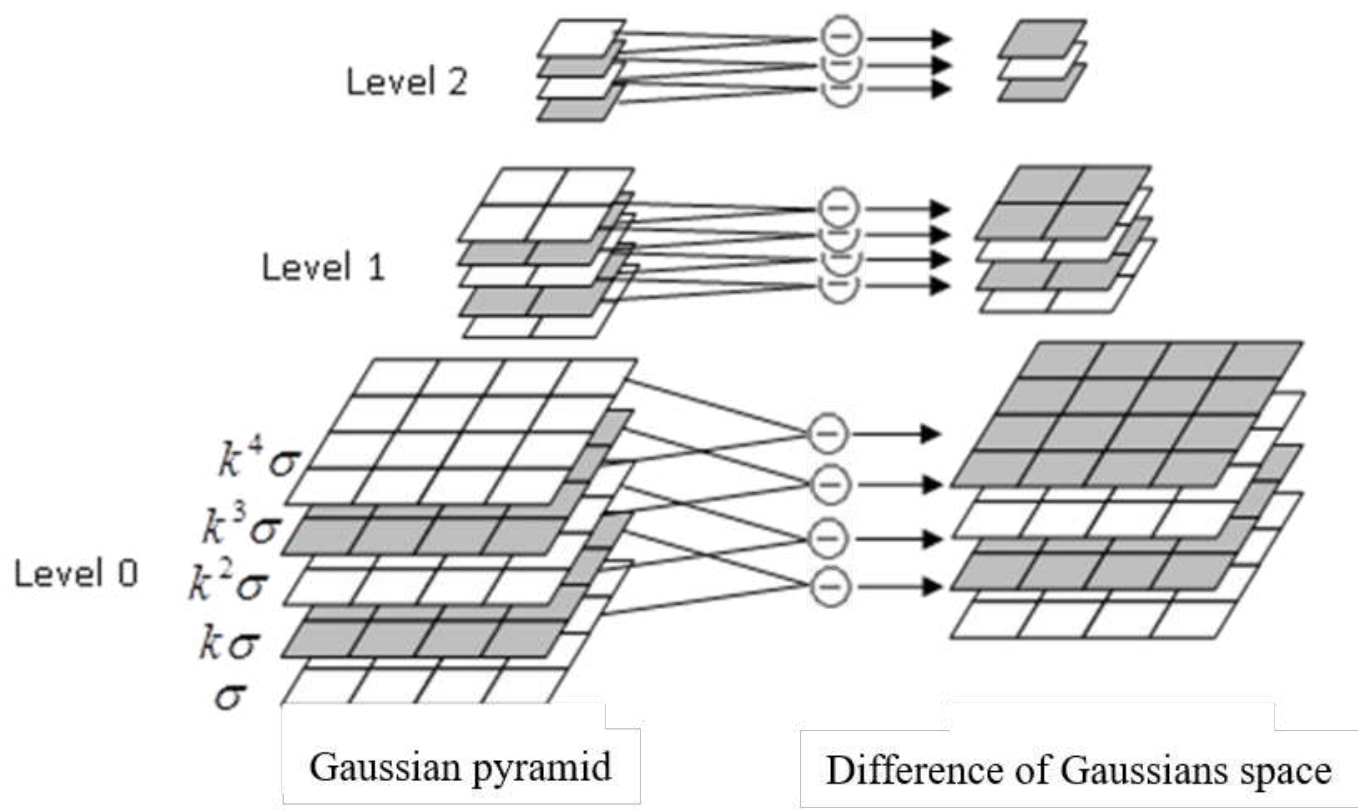

Fig. 7. Schematic for Difference of Gaussians space 
The local extreme point was detected in the Difference of Gaussians image and compared with a total of 26 pixels. The relative maximum or minimum was the local feature point of the image, as shown in Fig. 8.

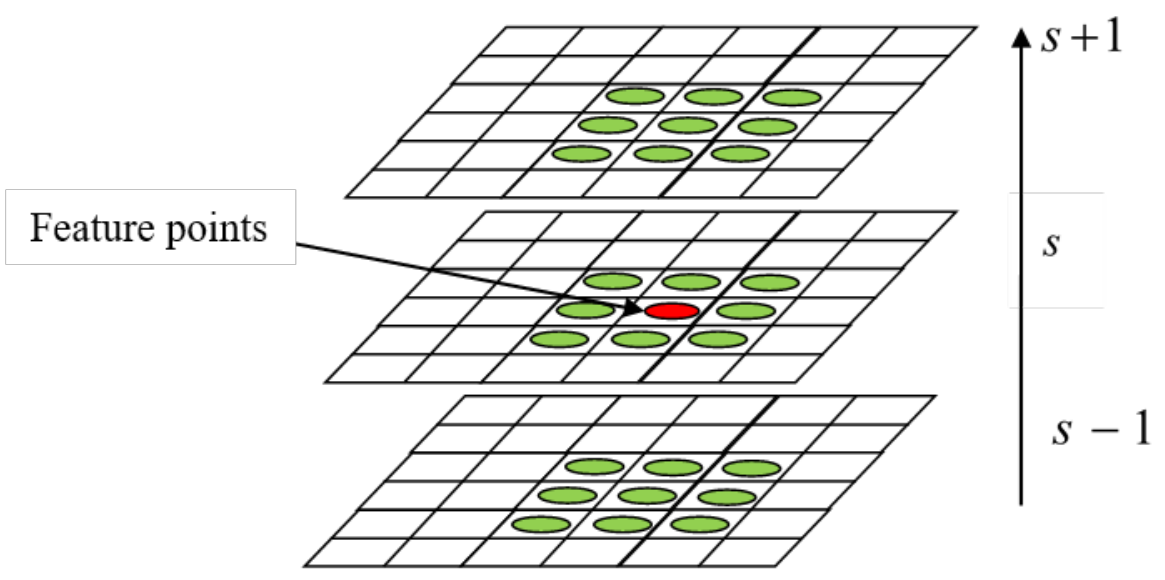

Fig. 8. Extraction of Difference of Gaussians feature points

The feature points extracted from the local feature points may include low-contrast feature points and boundary noise points. The scale space function $D(x, y, \sigma)$ was expanded into quadratic terms via Taylor expansion around the local extreme point $D_{0}$.

$$
\begin{aligned}
& D(x)=D_{0}+\frac{\partial D^{T}}{\partial x} x+\frac{1}{2} x^{T} \frac{\partial^{2} D}{\partial x^{2}} x \\
& \hat{x}=-\left(\frac{\partial^{2}}{\partial x^{2}}\right)^{-1} \frac{\partial D}{\partial x}
\end{aligned}
$$

where $x=x(x, y, \sigma)^{T}$ is the displacement of the local feature points, and is assumed to be 0 . In Eq. (8), $D(x)$ can be used to calculate the extreme $\hat{x}$ of the sample displacement. If the extreme is higher than threshold, the local feature points are regarded as unstable and removed, which can improve the stability and accuracy of the low-contrast feature point search. After the SIFT calculation on the LED chips, the SIFT feature point distribution was obtained as shown in Fig. 9. 


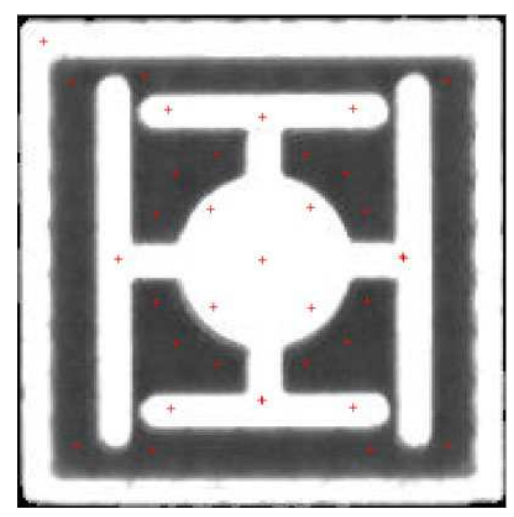

Fig. 9. SIFT feature point distribution

As the feature points were located in the grayscale blocks instead of at the pattern boundaries, if the grayscale in the area was different or defective, the system was more likely to make errors in the locating judgment, and it was not easy to reach the hierarchical location of each lithographic pattern. Therefore, the feature points were introduced to the Harris-Laplace feature point judgment method.

\subsubsection{Harris-Laplace feature point search method}

Both the Harris-Laplace algorithm and the SIFT algorithm adopt the Gaussian pyramid and Difference of Gaussians as the feature extraction space. Harris corner point detection was introduced into the K-level [11] to detect the boundary corner points in the level and match them to the coordinates in the K-1 layer. The boundary corner points detected at a higher level, i.e. the boundary corner points detected at a more blurred level, showed more stable feature points and higher calculation weights, as shown in Fig. 10.

Compared with the SIFT feature point comparison method, the feature points of the Harris-Laplace method were distributed at the boundary corner points of the lithographic patterns, which is similar to the case with the LED chip lithographic layered block. After the chips were calculated by the Harris-Laplace algorithm, the distribution of the Harris-Laplace feature points was as shown in Fig. 11. 


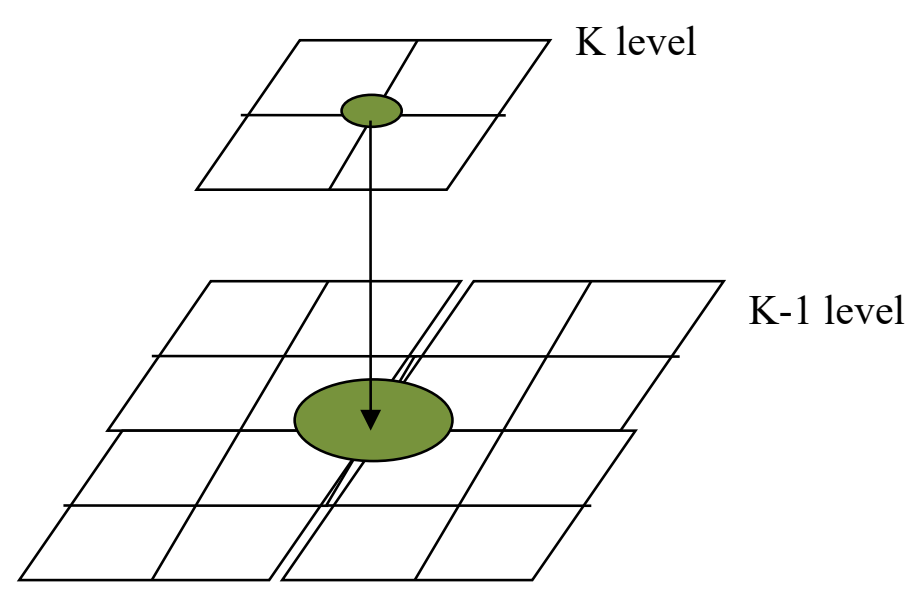

Fig. 10. Schematic for extraction of the Harris-Laplace feature points

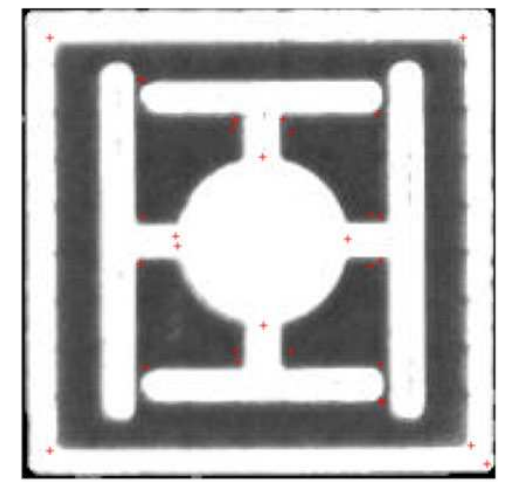

Fig. 11. Distribution of the Harris-Laplace feature points

\subsection{Feature area template locating method}

The matching step of the SIFT and Harris-Laplace methods requires two searches of the feature points, and the direction vector of the feature points must be calculated. The huge calculation workload increases the processing time. In the case of an image of size $180 \times 180$ pixels, the required comparison time is about $4.6 \mathrm{~s}$. To meet the requirements of industrial production lines, the Harris-Laplace method was adopted to train the feature areas, and then select the near trained feature points adjacent to the pixel areas as the feature area template, as shown in Fig. 12. In the figure, the dotted line is the light mask in the light-emitting area measured by the template, the black box is the light mask in the finger electrode area measured by the template, and the gray box is the light mask in the pad electrode area measured by the template. Feature area template comparison was then adopted to explore the relative location of the locating points between the templates and calculate the relative displacement and scale changes 
between the templates, which shortened the comparison time.

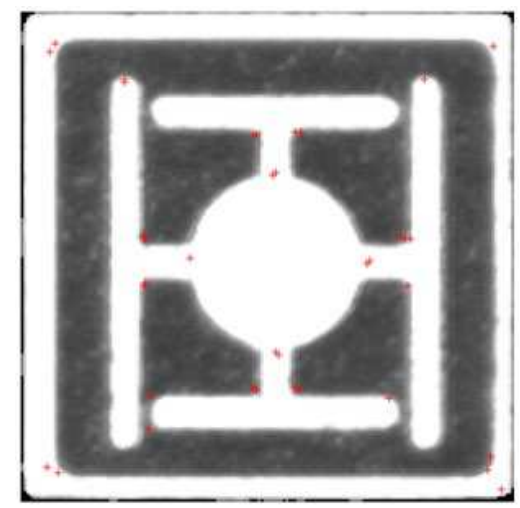

(a) Harris-Laplace feature point area

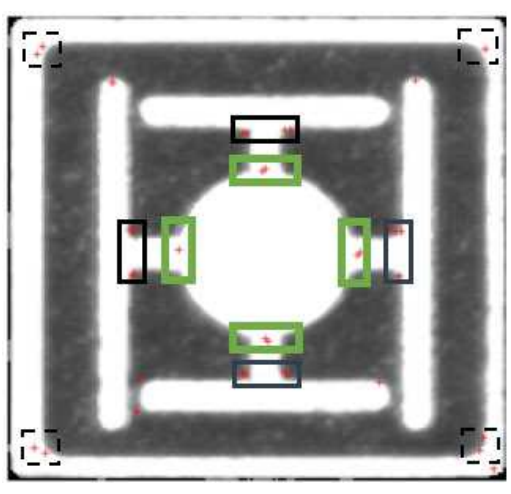

(b) Feature area template

Fig. 12. Feature area template

In its locating process, the feature area template was divided into $13 \times 13$ pixels and $13 \times 25$ pixels, and the centroid coordinates of each feature area template were regarded as the location of the corresponding feature area pattern. Then, the locating points on the LED chip patterns were determined through the feature template search, and the influencing parameters of the displacement and scale changes between the light masks were obtained. The pre-established standard template was adjusted based on the affine transformation of the influencing parameters. As shown in Figure 13(a)-(c), the standard template was adjusted to fit the locating points of each chip pattern area. Later, the adjusted standard template and the center reference point of the adaptive template were superimposed on the chip pattern so that the adaptive template fitted the chip pattern. The locating of the feature area template and the establishment of the adjusted adaptive template are shown in Fig. 13. The affine transformation operation is shown in Eq. (9).

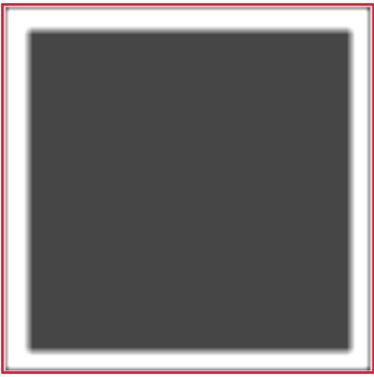

(a) Standard template for the light-emitting area

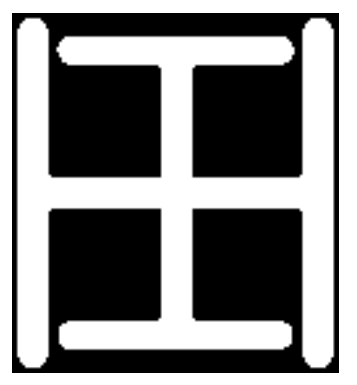

(b) Standard template for the finger electrode area

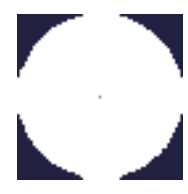

(c) Standard template for the pad electrode area 


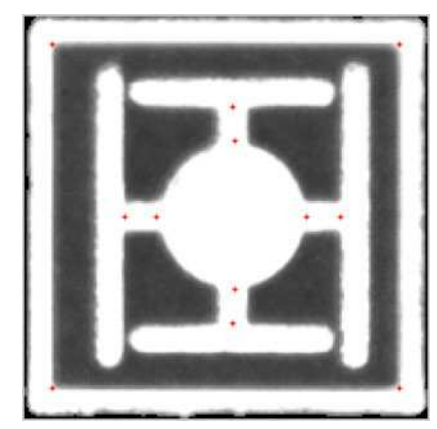

(d) Locating of the feature area template

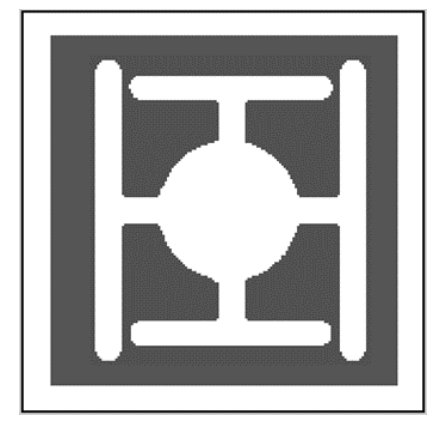

(e) Adaptive template

Fig. 13 Locating of the feature area template and establishment of the adaptive template

$$
\left[\begin{array}{l}
r^{\prime} \\
c^{\prime}
\end{array}\right]=\left[\begin{array}{ll}
a_{11} & a_{12} \\
a_{21} & a_{22}
\end{array}\right] \times\left[\begin{array}{l}
r \\
c
\end{array}\right]+\left[\begin{array}{l}
t_{r} \\
t_{c}
\end{array}\right]
$$

where $r$ and $c$ are the coordinates of the standard template, $a_{11}, a_{12}, a_{21}$, and $a_{22}$ are the conversion parameters, which can adjust the rotation or zoom of the images, $t_{r}$ and $t_{c}$ are the displacement parameters, $r^{\prime}$ and $c^{\prime}$ are the coordinates of the standard template converted to the adaptive template. The transformation nd translation parameters in the affine transformation were changed to translate, scale, and rotate the images according to the locating point information, and to establish the adaptive template.

\subsection{Optimal template boundary fitting}

During the fitting process of the adaptive template, a poor fitting occurs at the boundaries if the adaptive template is directly compared to the LED chip and template, as shown in Fig. 14. This is because the chip lithographic process leads to inconspicuous contrast at the chip lithographic pattern boundary. Therefore, a Gaussian blur function was used to smooth the boundaries of the established adaptive template and set the binarization threshold to achieve the optimal template boundary fitting effect. 


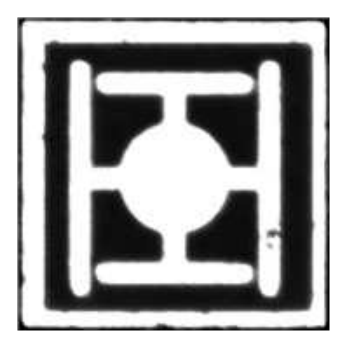

(a) Original image

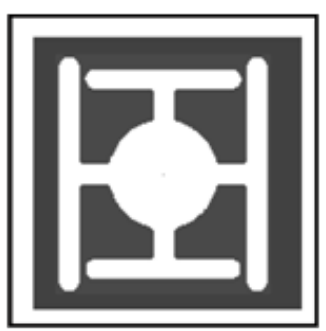

(b) Adaptive template

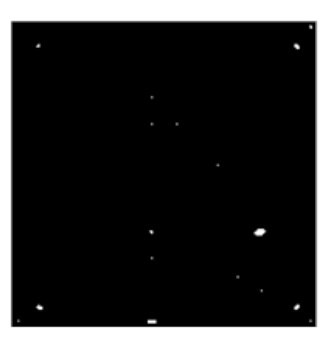

(c) Defect segmentation

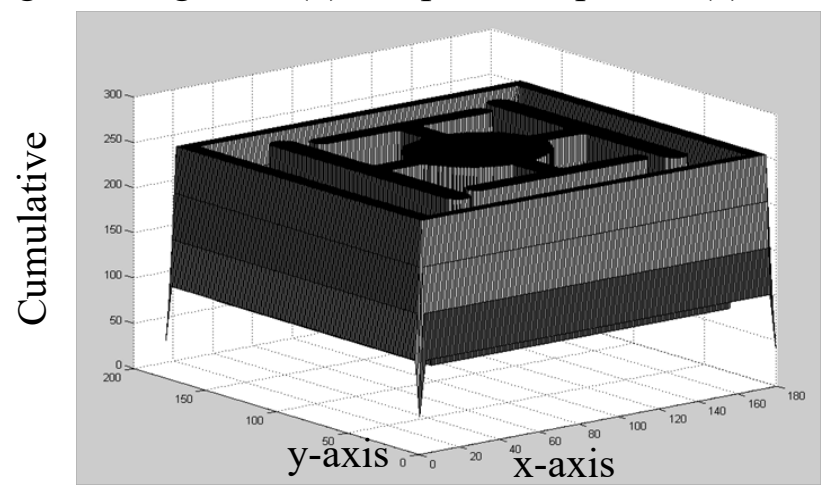

(d) Adaptive template and grayscale distribution

Fig. 14 Adaptive template not blurred

A 13-pixel line-width was used as the standard template for the finger electrode area, and the adaptive template was blurred by a Gaussian blur function of dimensions $3 \times 3$ pixels, as shown in Fig. 15. The fitted adaptive chip template was subtracted from the original chip image to extract the defects, as shown in Fig. 16(a). Then, the multiarea growth method was used to separate the defects into different pattern areas so that the system could judge the defects (see Figs. 16(c)-(e)). 


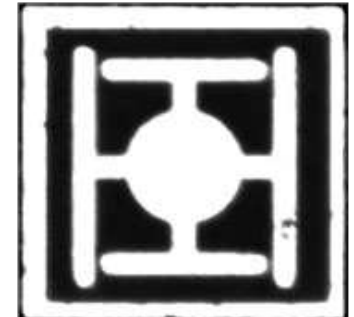

(a) Original image

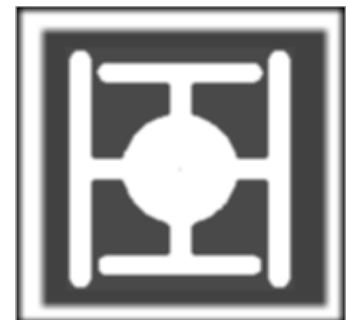

(b) Adaptive template

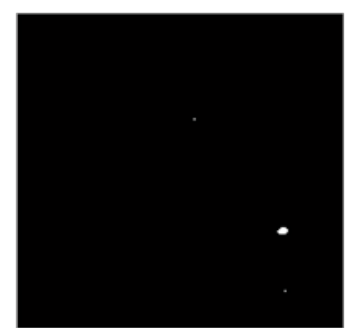

(c) Defect segmentation

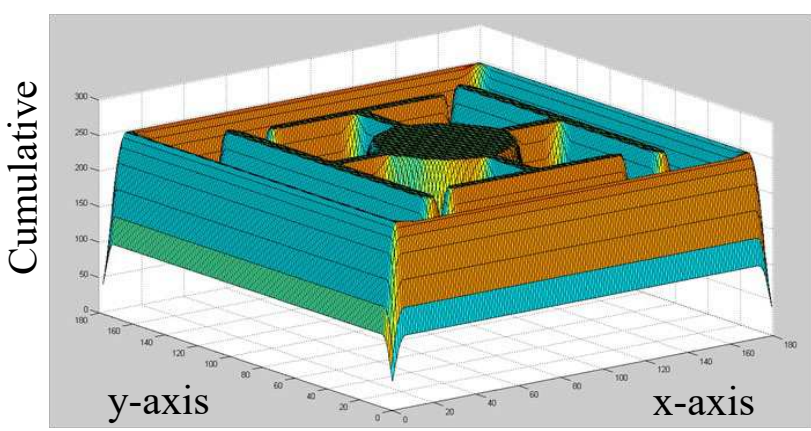

(d) Adaptive template and grayscale distribution

Fig. 15. Fitting and blurring of the adaptive template

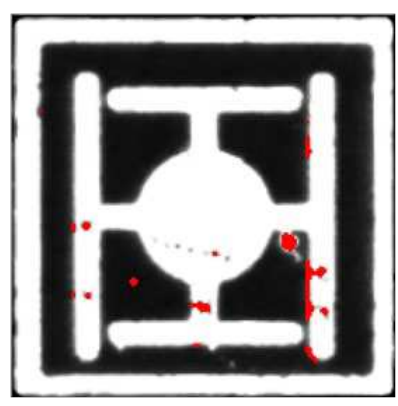

(a) Defect distribution

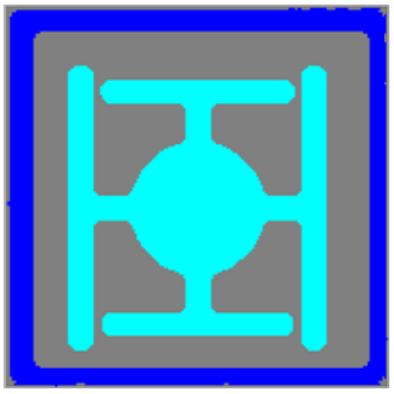

(b) Lithographic area

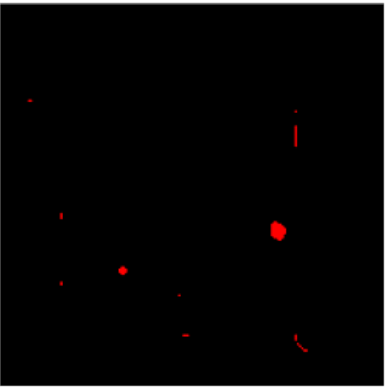

(d) Light-emitting area

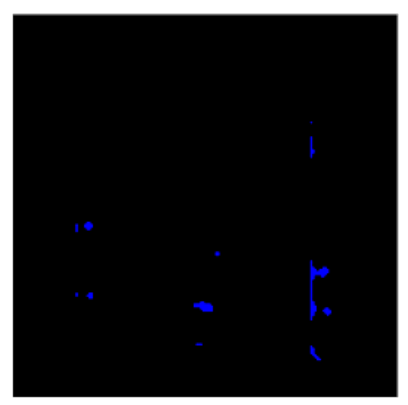

(e) Electrode area

(c) Mesa area

Fig. 16. Defect extraction and partitioning

The overall process in this study was divided into two stages, as shown in Fig. 17. The first was the image preprocessing stage. Here, the wafer image was segmented to 
obtain the chip sub-images, and the chip pattern integrity was fast-screened. The second was the adaptive template establishment stage. In this stage, the feature points were located quickly through the screened chip image, and the adaptive template was established. Finally, the image defects were extracted to assist the user in detection.

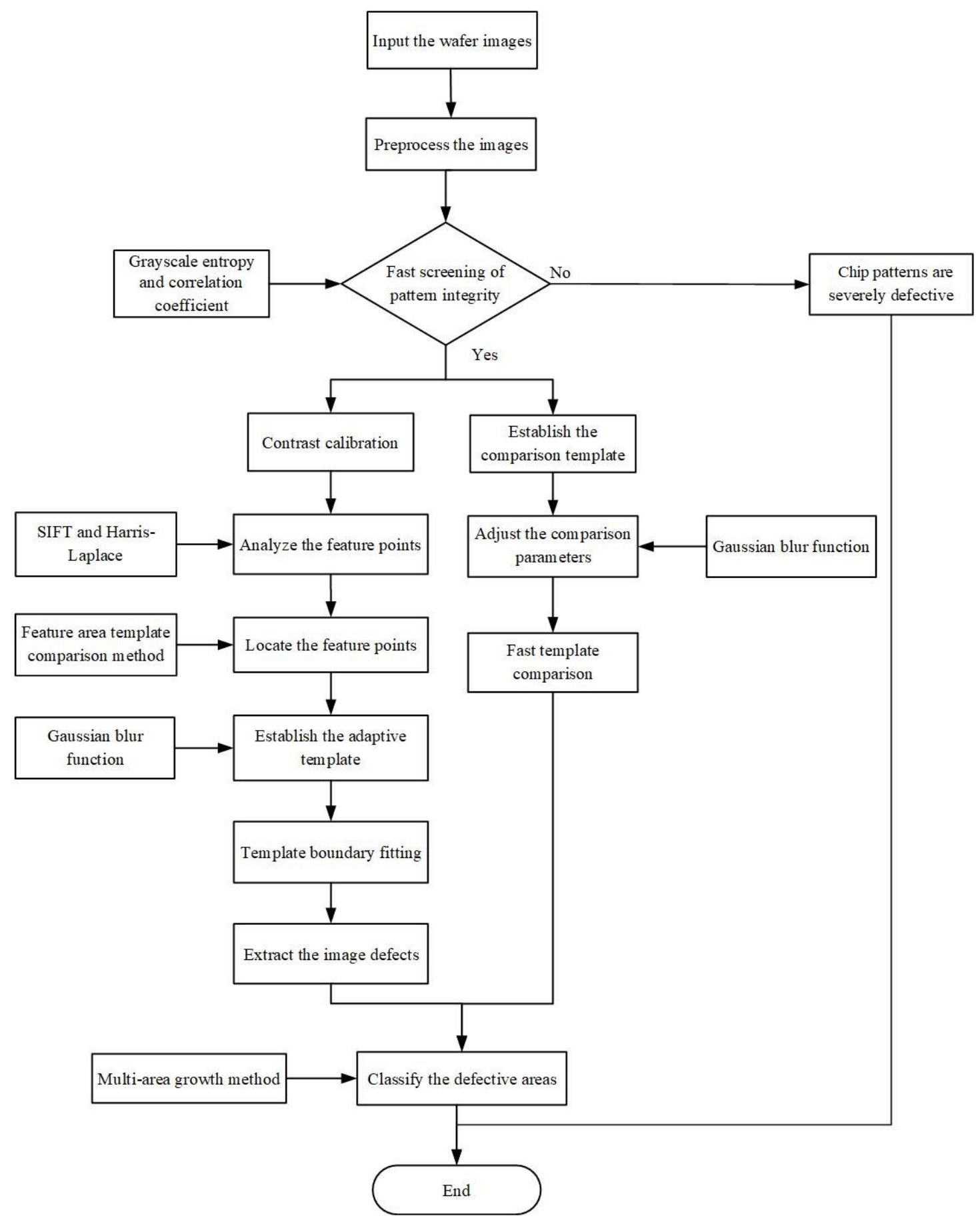

Fig. 17. Detection process

\section{Experimental details}


The chip sample in this study was the LED wafer images captured by the LED detector shown in Fig. 18(a). These LED wafer images were divided into LED chip sub-images through digital image processing, as shown in Fig. 18(b). The LED chip sample used included the finger electrode area, pad electrode area, light-emitting area, and mesa area, as shown in Fig. 18(c). The detection specifications for this chip are shown in Table 1. Specifically, the light gray stripe is the finger electrode area, the middle circle is the pad electrode area, the gray background is the light-emitting area and the peripheral white area is the mesa area.

Table 1 Surface detection specifications for LED chips

\begin{tabular}{|c|c|c|c|c|}
\hline $\begin{array}{l}\text { Defective } \\
\text { area }\end{array}$ & Importance & $\begin{array}{l}\text { Name of } \\
\text { defect }\end{array}$ & Illustration & Remark \\
\hline $\begin{array}{l}\text { Electrode } \\
\text { area }\end{array}$ & Major & Metal loss & & $\begin{array}{l}\text { 1. The area of pad metal loss was } \\
\text { larger than } 130 \text { pixels } \\
\text { 2. Metal loss or line breakage in the } \\
\text { finger }\end{array}$ \\
\hline $\begin{array}{l}\text { Electrode } \\
\text { area }\end{array}$ & Major & Residual loss & & $\begin{array}{l}\text { The area of residual metal was } \\
\text { larger than } 130 \text { pixels }\end{array}$ \\
\hline $\begin{array}{l}\text { Electrode } \\
\text { area }\end{array}$ & Minor & Contamination & & $\begin{array}{l}\text { The area of pad contamination was } \\
\text { larger than } 130 \text { pixels }\end{array}$ \\
\hline $\begin{array}{l}\text { Light- } \\
\text { emitting } \\
\text { area }\end{array}$ & Major & $\begin{array}{l}\text { Dirt and } \\
\text { damage }\end{array}$ & & $\begin{array}{l}\text { 1. The area of dirt was larger than } \\
50 \text { pixels } \\
\text { 2. The area of damage was larger } \\
\text { than } 50 \text { pixels }\end{array}$ \\
\hline $\begin{array}{l}\text { Light- } \\
\text { emitting } \\
\text { area } \\
\text { Mesa }\end{array}$ & Minor & $\begin{array}{l}\text { Boundary } \\
\text { abnormality }\end{array}$ & & $\begin{array}{l}\text { 1. Boundaries were not complete } \\
\text { 2. The area of dirt on the boundaries } \\
\text { was larger than } 180 \text { pixels }\end{array}$ \\
\hline $\begin{array}{l}\text { Damaged } \\
\text { chips }\end{array}$ & Major & $\begin{array}{l}\text { Damaged } \\
\text { chips }\end{array}$ & & $\begin{array}{l}\text { Edge collapse or cut of the light- } \\
\text { emitting area }\end{array}$ \\
\hline
\end{tabular}




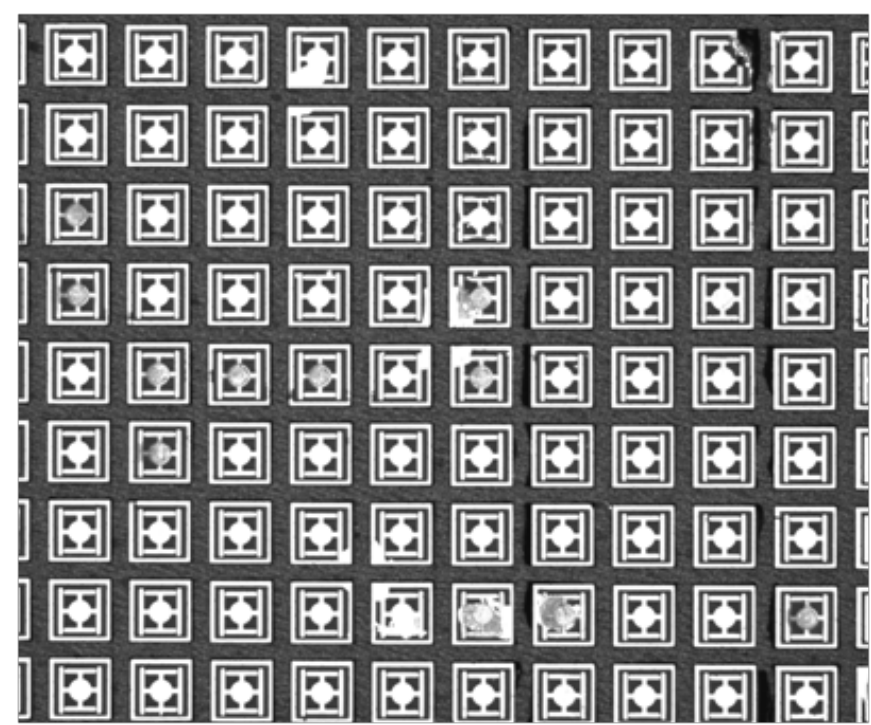

(a) Wafer image

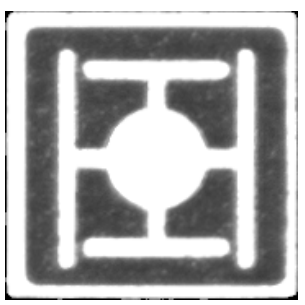

(b) Chip sub-image

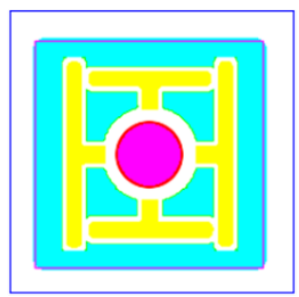

(c) Chip layer

Fig. 18. LED chip sample used in this study

\subsection{Image extractor and measurement system}

The LED wafer image samples used in this study were extracted by the selfdesigned automated optical detector shown in Fig. 19. The relevant shooting environments and parameters are shown in Table 2.

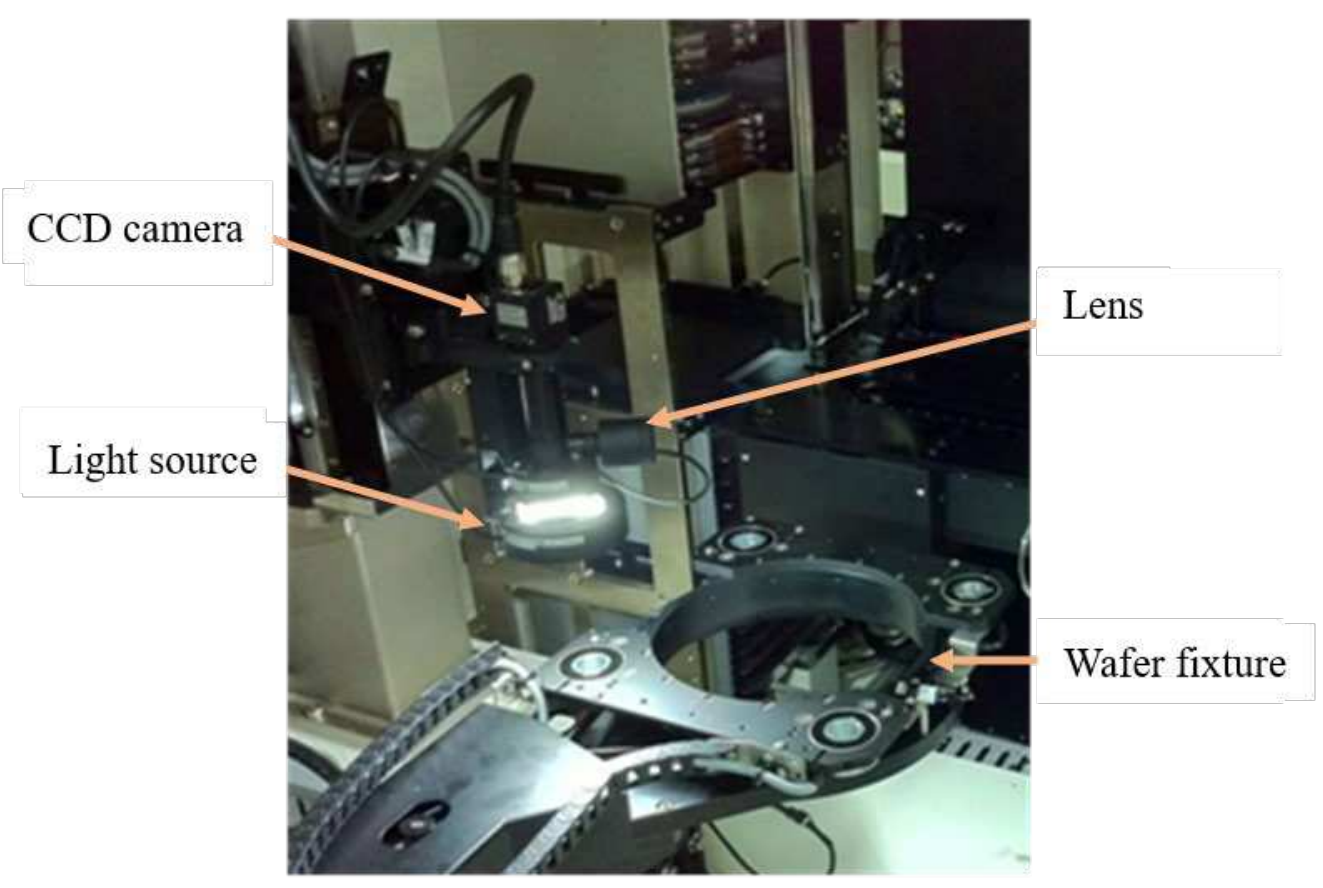

Fig. 19. Detector system

Table 2 Experimental environmental parameters 


\begin{tabular}{|l|l|}
\hline $\begin{array}{l}\text { Shooting } \\
\text { environment }\end{array}$ & Environmental parameters \\
\hline Detected object & LED 4-inch wafer \\
\hline CCD camera & IMPER ICDA-IPX-B2520-L \\
\hline Camera resolution & $2456 \times 2058$, Mono 10-bit \\
\hline $\begin{array}{l}\text { Camera extraction } \\
\text { frequency }\end{array}$ & $16 \mathrm{fps}$ \\
\hline Lens & Fixed $2 \mathrm{X}$ lens \\
\hline Pixel size & $1.75 \mu \mathrm{m}$ \\
\hline Light source & Circular white light source and coaxial white light source \\
\hline
\end{tabular}

\subsection{Image preprocessing}

Fig. 20 shows the steps involved in the image preprocessing. In the image preprocessing, the wafer images extracted by the automated detector were analyzed. First, the wafer images extracted by the system were median-filtered to eliminate the background blue film texture of the back wafer and avoid excessive noise in subsequent processing. Then, the filtered images were subjected to the Sobel edge calculation to completely select the chip boundary. The morphological opening and closing operations were performed to intensify the chip boundary images and eliminate the tiny image noises. Next, the area was filled according to the chip boundaries to form the LED wafer shield and facilitate the extraction of wafer sub-images. Depending on the connection marks, the convolution integral was evaluated on the LED wafer shield and the original images to extract the chip images. The extracted chip frame was externally rotated to ensure that the extracted chip had no frame rotation variables. 


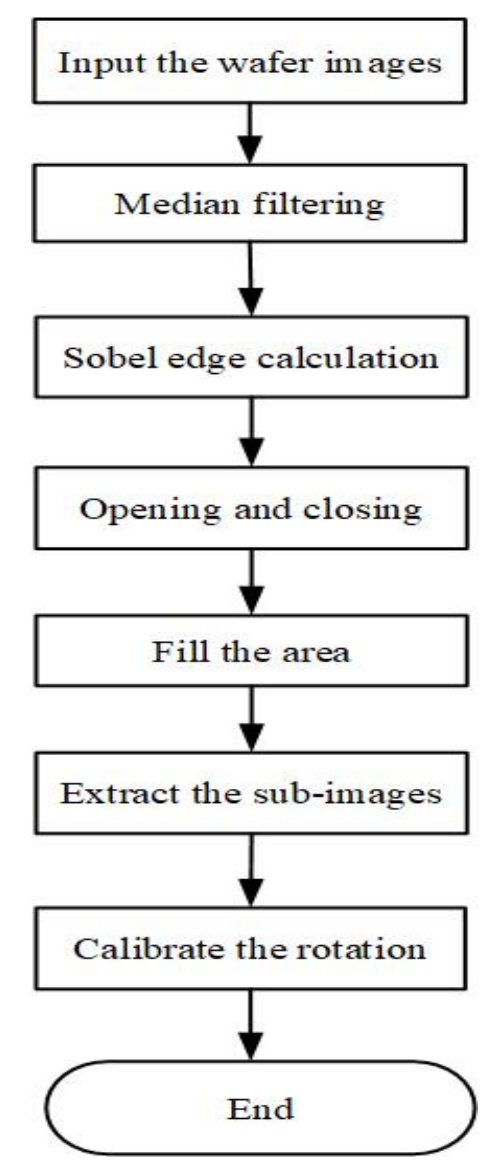

Fig. 20. Image preprocessing

\subsection{Adaptive template comparison}

Fig. 21 shows the adaptive template comparison process of this study. The comparison and correction aimed to stretch the contrast of the fast-screened chip images to intensify the chip boundaries. In this study, the Harris-Laplace feature areas of the chips were located using the feature template method. According to the feature area template method, the feature points of the input chips were located, while the relative positions of the feature points were used to discuss the variables in each layer. Next, the adaptive template was adjusted according to the variables to fit the chips. Then, the boundaries of the adaptive template were fitted using a Gaussian blur function to reach an optimal template comparison effect and extract the image defects. 


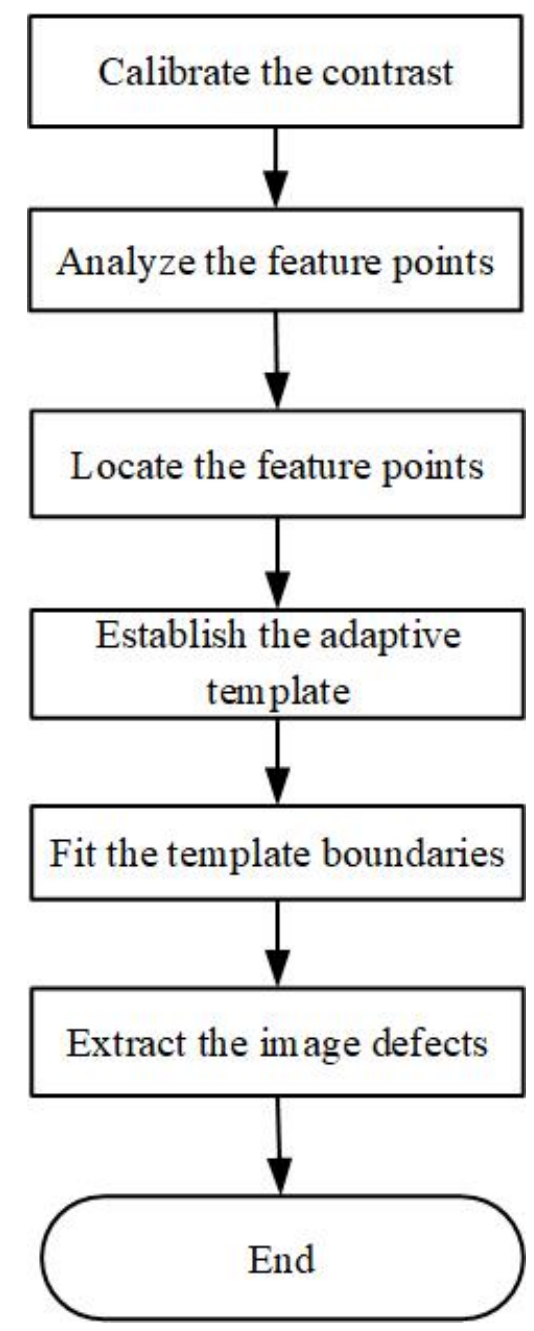

Fig. 21. Adaptive template comparison process

\subsection{Receiver operating characteristic curve}

The receiver operating characteristic (ROC) curve [43][44] was used in this study for overall evaluation and verification. Using the confusion matrix (see Table 3 ) and the related ROC equations (see Eqs. (10)-(12)), the judgment of the dominant and recessive samples by the system could be displayed and the judgment results summarized by the ROC curve.

Table 3 Confusion matrix

\begin{tabular}{|c|c|c|}
\hline \multirow{2}{*}{ System results } & \multicolumn{2}{|c|}{ Actual results } \\
\cline { 2 - 3 } & $T P$ & $F P$ \\
\hline Total & $F N$ & $T N$ \\
\hline
\end{tabular}


The related ROC equations are as follows:

$T P R=\frac{T P}{T P+F N}$

$F P R=\frac{F P}{F P+T N}$

$A C C=\frac{T P+T N}{P+N}$

where $\mathrm{P}$ is the total number of positive samples, $\mathrm{N}$ is the total number of negative samples, TP (true positive) is the number of actually positive samples that were judged as positive, TN (true negative) is the number of actually negative samples that were judged as negative, FN (false negative) is the number of actually positive samples that were judged as negative, FP (false positive) is the number of actually negative samples that were judged as positive, TPR (true positive rate) is the ratio of TP samples to all positive samples, FPR (false positive rate) is the ratio of FP samples to all negative samples, and ACC (accuracy) is the overall system correction rate.

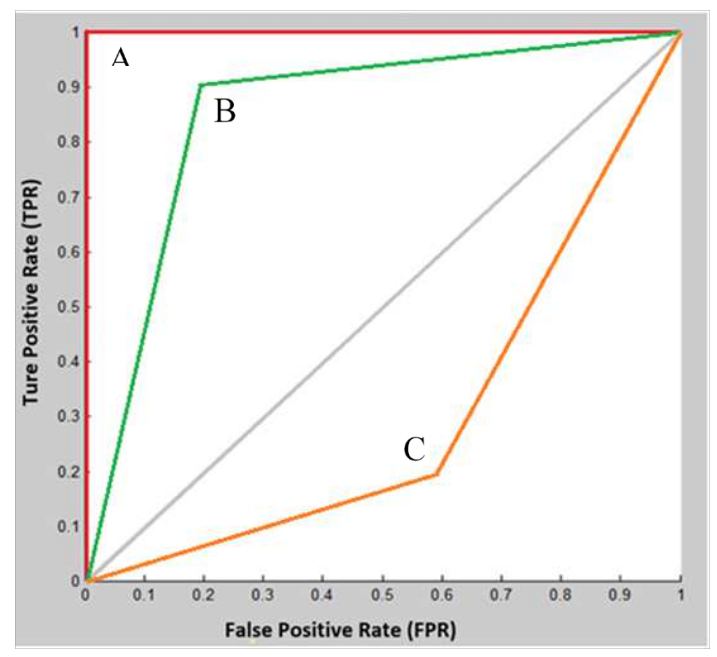

Fig. 22. Schematic of the ROC curve

The system ROC curve can be obtained from the above parameters as shown in Fig. 22, where the horizontal axis is the FPR and the vertical axis is the TPR. If the judgment results are closer to the upper left, the system judgment accuracy is higher; if the judgment results are closer to the lower right corner, the system judgment accuracy is lower. The diagonal line is the zero recognition line. If the judgment results are on 
the diagonal line, the system has no recognition ability. Among curves A, B, and C in the figure, the system represented by curve $A$ has the best judgment effect, followed by the system represented by curve B. Curve $\mathrm{C}$ is lower than the zero recognition line, which indicates that the probability of incorrect judgment of the system represented by curve $\mathrm{C}$ is higher than the probability of correct judgment. As ROC cannot be used as a binary recognition system, the advantages and disadvantages of the system can be quickly resolved by the ROC curve.

\subsection{Training of the grayscale entropy screening threshold during fast screening}

Before the adaptive template was established, the severely damaged chips were removed, through fast screening of the chip pattern integrity, to improve the locating accuracy of the feature points. During rapid screening, the grayscale entropies of the defective and non-defective chips were very different. Therefore, the index threshold was selected based on the non-defective chips, and all the LED chips were described according to the definition of defects in Table 1. A total of 50 non-defective chips were selected for analysis, and the six sigma was adopted to select the threshold.

The grayscale entropy $€$, total average, and standard deviation of 50 groups of nondefective chip samples were calculated. According to the definition of six sigma [45][46], the total average and the total average \pm six sigma were used to select the screening thresholds. The six sigma could reach $99.99966 \%$ of the total number of samples. In this study, the six sigma covered all the non-defective chips, as shown in Fig. 23 and expressed by Eqs. (13)-(17)

$$
\begin{aligned}
& E=\sum_{i=100}^{210}-p(i) \times \log p(i) \\
& \mu=\frac{1}{50} \sum_{i=0}^{50} E(i)
\end{aligned}
$$

$$
\sigma=\sqrt{\frac{1}{50} \sum_{i=1}^{50}(E(i)-\mu)^{2}}
$$

$$
T_{u p}=\mu+6 \sigma
$$

$$
T_{\text {down }}=\mu-6 \sigma
$$

In this study, the grayscale entropy range of the trained 50 groups of non-defective 
chips was calculated, and the results are shown in Table 4. The grayscale entropy indices of all the 364 LED chips were screened, including 225 non-defective chips and 139 defective chips. The results are shown in Figs. 24 and 25. Based on the grayscale entropy index, 40 severely damaged chips were removed.

Table 4 Grayscale entropy index

\begin{tabular}{|c|c|c|c|c|}
\hline & $\begin{array}{c}\text { Grayscale entropy } \\
\text { average } \mu\end{array}$ & $\begin{array}{c}\text { Grayscale entropy } \\
\text { standard deviation } \\
\sigma\end{array}$ & $T_{u p}$ & $T_{\text {down }}$ \\
\hline $\begin{array}{c}50 \text { groups } \\
\text { of chips }\end{array}$ & 24685.52 & 1702.05 & 34897 & 14473 \\
\hline
\end{tabular}

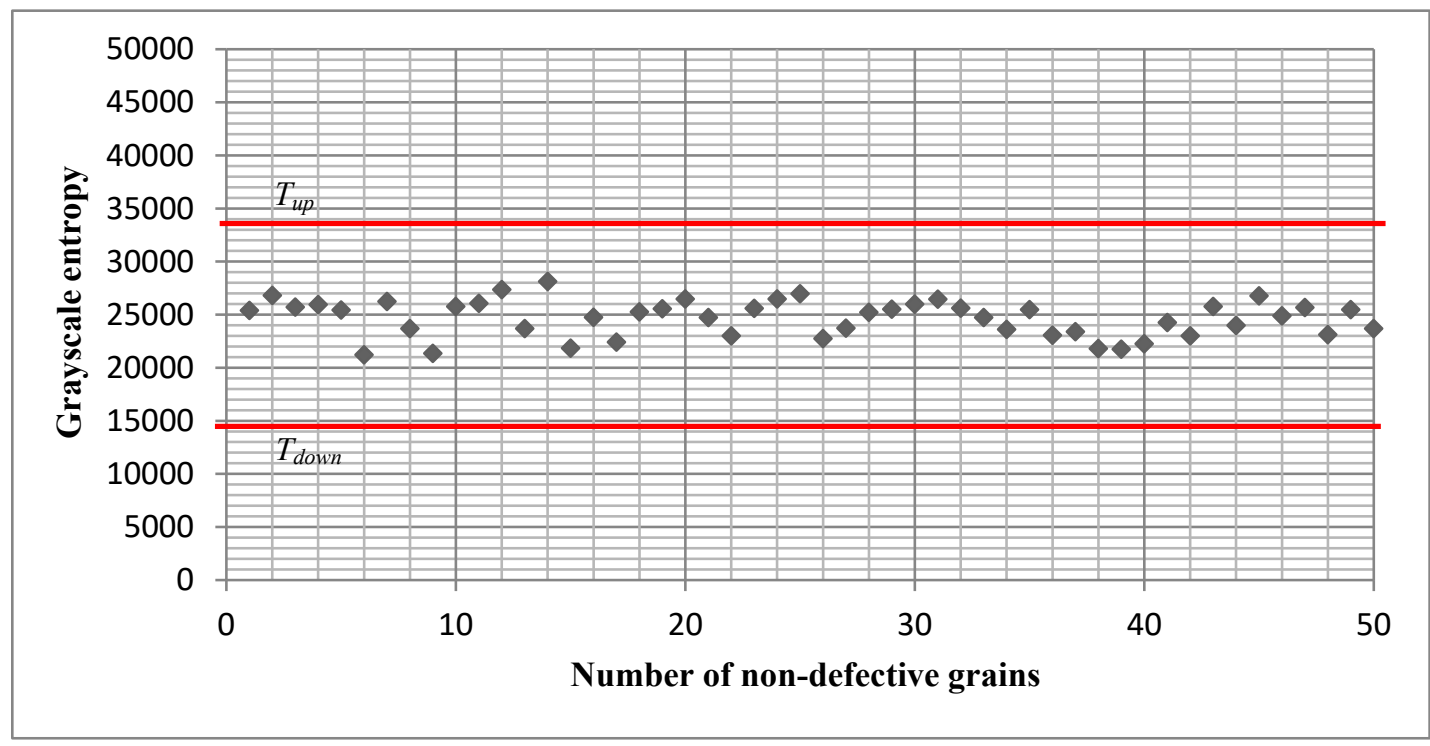

Fig. 23. Distribution of the grayscale entropy of 50 groups of non-defective chips

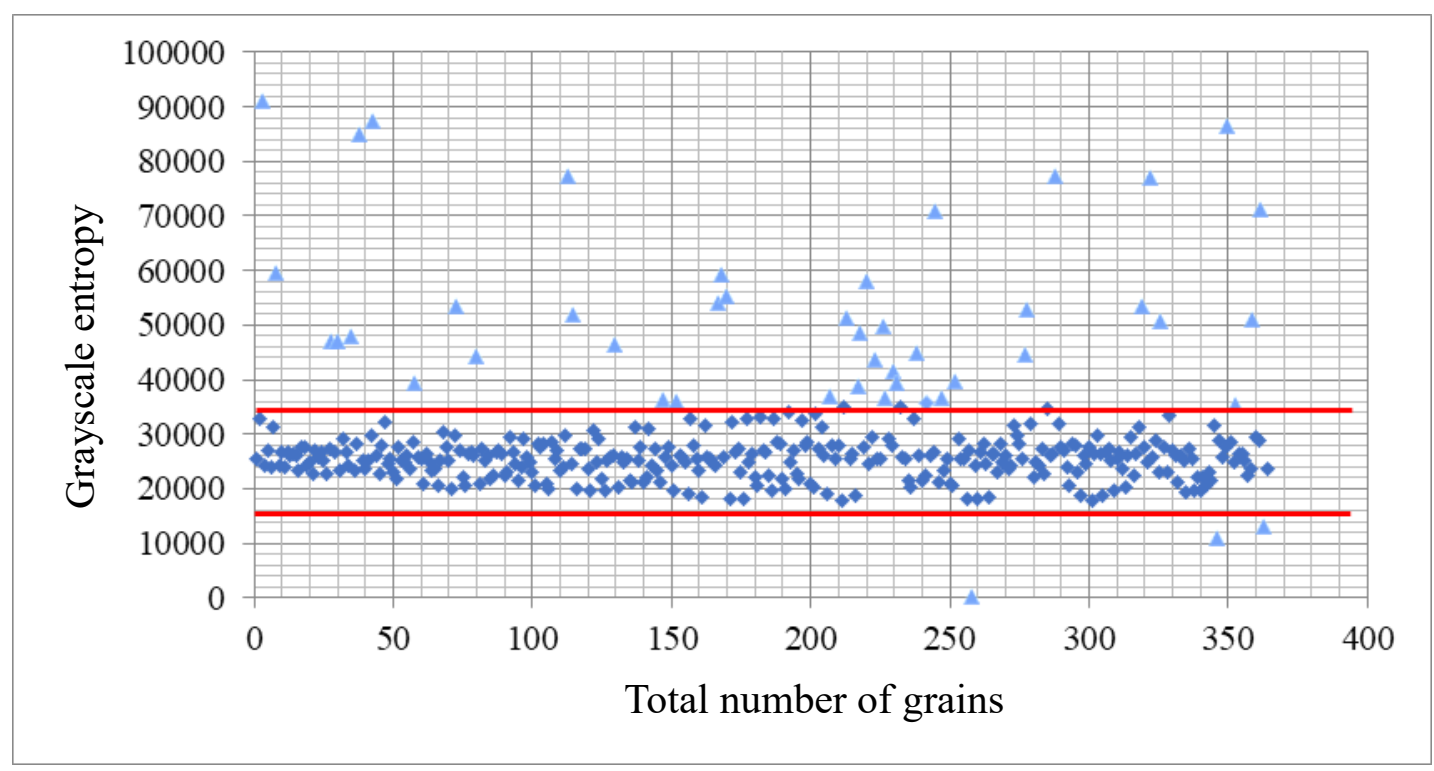


Fig. 24. Distribution of the grayscale entropy of all the chips
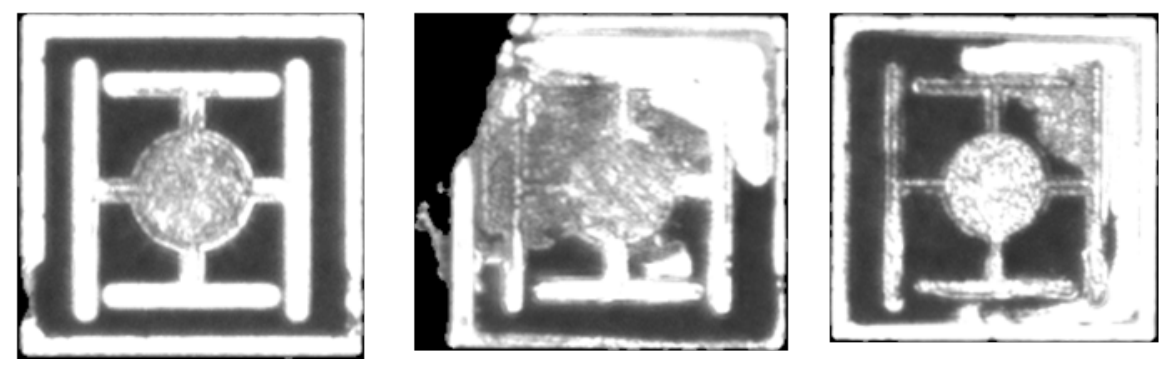

Fig. 25. Severely damaged chips

\subsection{Training of the correlation coefficient screening threshold during fast screening}

In this study, the correlation coefficient index was used to judge whether the chips were defective or not. During the screening of the correlation coefficient index, the ROC parameters were used to select the thresholds. During the extraction of training samples, as shown in Fig. 26, all the LED chip samples were randomly sampled, and the No. 1 to No. 100 chips were trained subsequently. The defects of the 100 chips were detected according to the detection specifications in Table 1. The defect detection results were compared with the defect detection results of the screened chips under different correlation coefficients to obtain the correct judgment, leakage, and overkill phenomenon. The highest ACC was 0.75 , and the correlation coefficient threshold was set to 0.92, as shown in Table 5. The defective pattern is shown in Fig. 27.

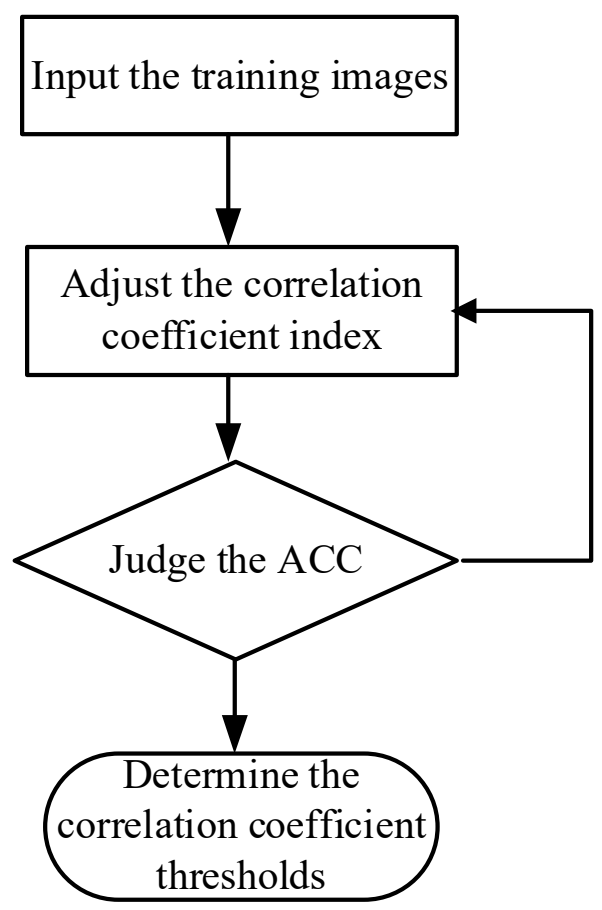


Fig. 26. Screening process for the correlation coefficient thresholds

Table 5 Screening rules for the correlation coefficients

\begin{tabular}{|c|c|c|c|c|c|c|c|c|c|c|c|c|}
\hline $\begin{array}{c}\text { Correlation } \\
\text { coefficient } \\
\text { index }\end{array}$ & $\mathbf{0 . 8 5}$ & $\mathbf{0 . 8 6}$ & $\mathbf{0 . 8 7}$ & $\mathbf{0 . 8 8}$ & $\mathbf{0 . 8 9}$ & $\mathbf{0 . 9}$ & $\mathbf{0 . 9 1}$ & $\mathbf{0 . 9 2}$ & $\mathbf{0 . 9 3}$ & $\mathbf{0 . 9 4}$ & $\mathbf{0 . 9 5}$ & $\mathbf{0 . 9 6}$ \\
\hline $\begin{array}{c}\text { Correct } \\
\text { judgment }\end{array}$ & 69 & 70 & 70 & 70 & 71 & 73 & 73 & 75 & 74 & 70 & 64 & 46 \\
\hline Leakage & 31 & 30 & 30 & 30 & 29 & 27 & 27 & 25 & 25 & 20 & 13 & 0 \\
\hline Overkill & 0 & 0 & 0 & 0 & 0 & 0 & 0 & 0 & 1 & 10 & 18 & 54 \\
\hline$A C C$ & 0.69 & 0.7 & 0.7 & 0.7 & 0.71 & 0.73 & 0.73 & 0.75 & 0.74 & 0.7 & 0.64 & 0.46 \\
\hline
\end{tabular}

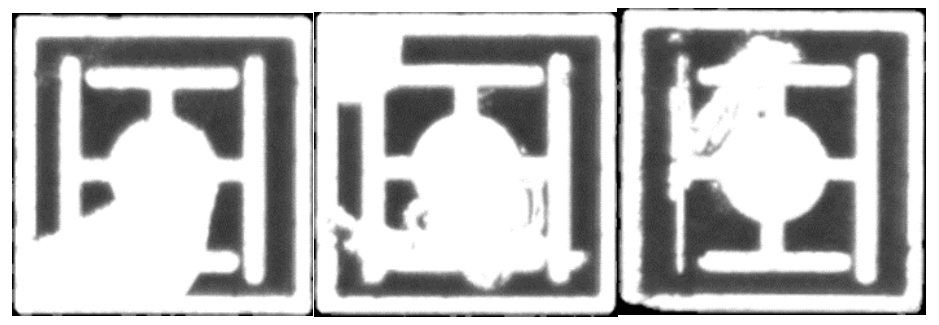

Fig. 27. Defective chips screened by their correlation coefficient indices

\subsection{Impact of fast screening on location of the adaptive template}

All the LED chip samples were fast-screened based on the grayscale entropy index interval [14473, 34897], and the correlation coefficient index after training was 0.92. However, for each of the 364 chips, it took 9.951 s to read the images and statiscally deduce the grayscale entropy index, $8.677 \mathrm{~s}$ to calculate the correlation coefficient index, and only $0.052 \mathrm{~s}$ on average to screen one chip. After fast screening, the system removed 60 severely defective chips out of the 364 chips, and no overkill occurred.

The 60 severely defective chips were fast-screened and then confirmed by location of the feature area template. Next, the impact of the chips on location of the adaptive template was reconfirmed, of which only No. 46 chip could be located through the auxiliary locating points. As shown in Figs. 28(a) and (b), the locating points in the upper right corner of the mesa area were affected by the defective block, resulting in poor location. However, as location of the mesa area template was aided by the locating points in the lower right and upper left corners, the establishment of the adaptive template was not affected. The remaining screened chips could not be accurately 
positioned. In Figs. 29(d) and (e), the chips could not be located due to severe defects. Therefore, fast screening can greatly improve the locating accuracy of adaptive template positioning. Finally, the adaptive template method was used to establish the adaptive template and extract the defects in the 304 chips.

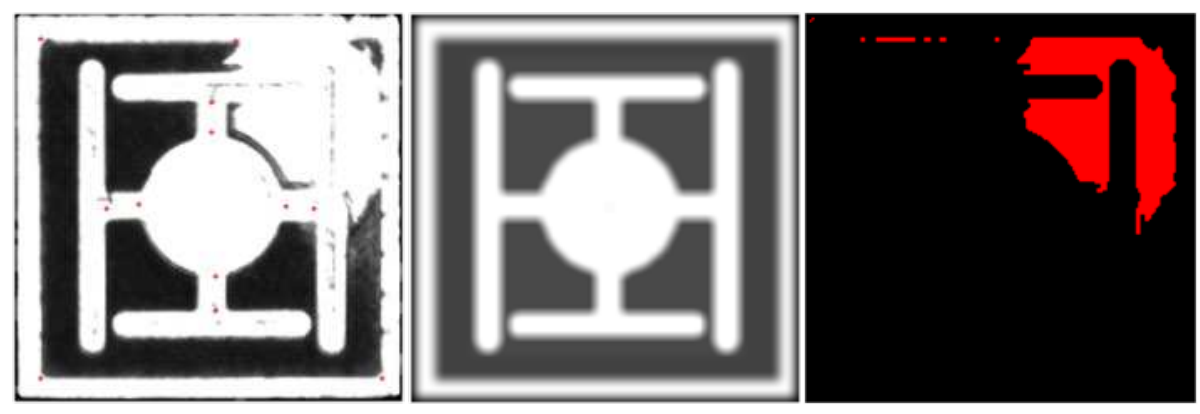
(a) Image after location
(b) Adaptive template
(c) Extraction of defective areas

Fig. 28. Establishment of the adaptive template based on fast-screened chips

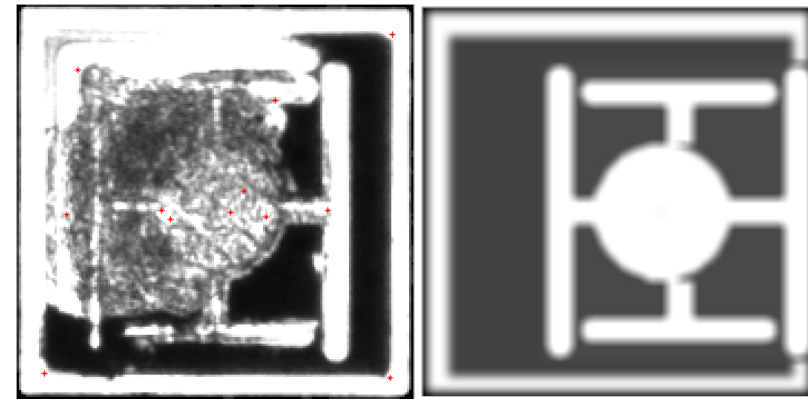

(d) Image after location

(e) Image after location

Fig. 29. Location confirmation for fast-screened chips

\subsection{Location results of the adaptive template}

By searching the feature area template, the locating points of the chip patterns were obtained to establish the adaptive template and fit the LED chip patterns. The sizes of the feature area template were $13 \times 13$ pixels and $13 \times 25$ pixels. When the adaptive template was actually established and the defects were extracted, it took about $0.14 \mathrm{~s}$ to establish a chip and about $0.11 \mathrm{~s}$ to extract the defects. Therefore, it took about $0.25 \mathrm{~s}$ in total to establish the adaptive template and extract the defects.

The layered areas of the LED chips were located by 12 locating points when the template was established. Specifically, there were three layered areas each located by four locating points located by four locating points respectively; the areas included the pad electrode area, finger electrode area, and light-emitting area. During the experiment, as some chip defects were located in the feature area template, the template locating 
center was set as the reference point, when the layered areas were located using the standard template, to avoid poor template location; this is shown in Fig. 30.

In the pad electrode area, four locating points were used to locate the layered areas, as shown in Fig. 31(a). The displacement of the upper locating points affected by defects was determined according to the locating points and the reference point. When only one locating point was affected, the remaining three locating points and the reference point were used to locate the adaptive template, as shown in Fig. 31 (b). The relationship between the locating points and the reference point in the finger electrode area and in the light-emitting area was the same, as shown in Figs. 32 and 33. The overall locating system could be more stable during the correction process of the locating points.

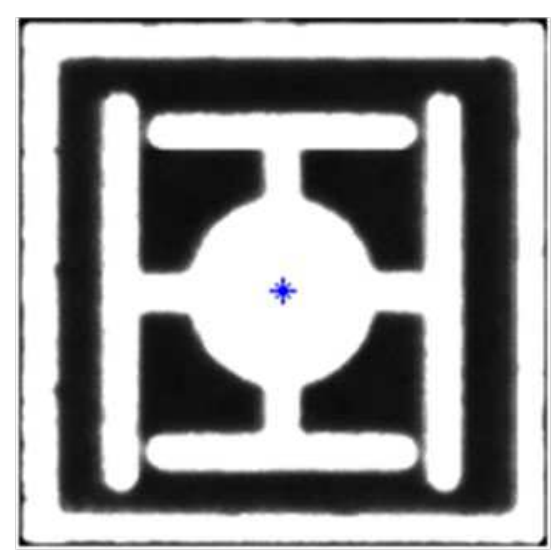

Fig. 30. Template locating reference point
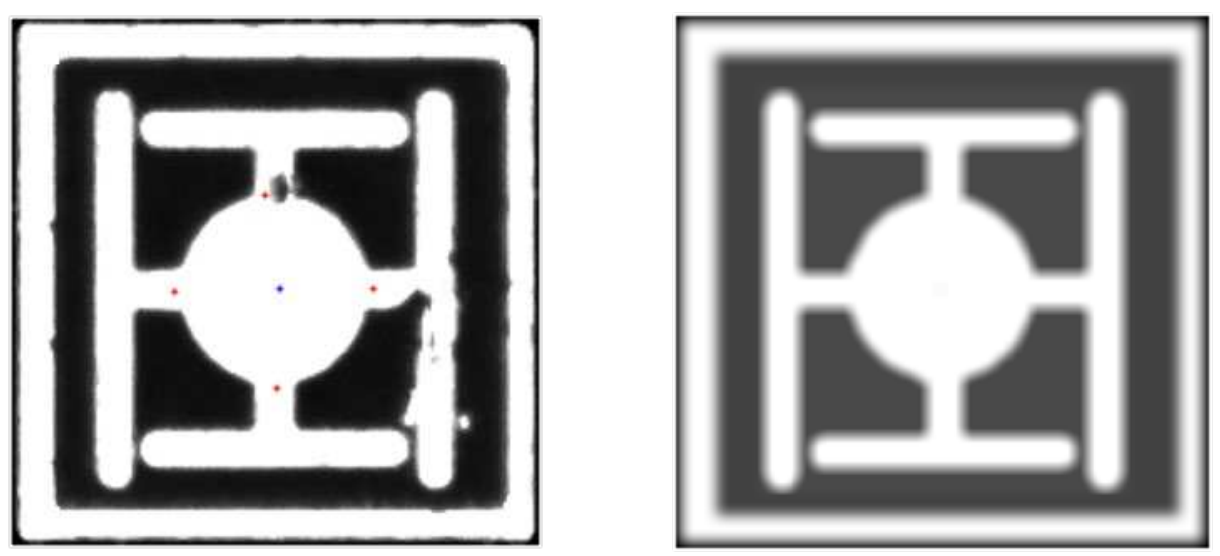

(a) Displacement of locating points in the pad electrode area

(b) Template with displacement corrected 
Fig. 31. Displacement of locating points in the pad electrode area and its correction

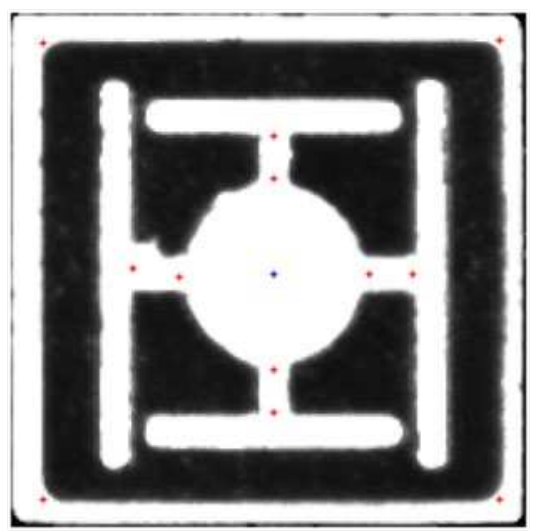

(a) Displacement of locating points in the finger electrode area

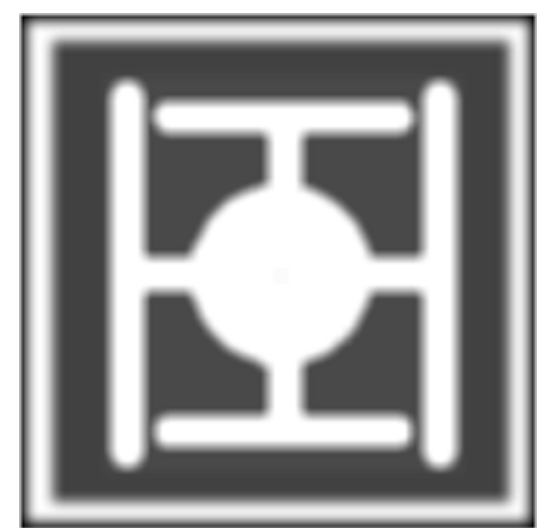

(b) Template with displacement corrected

Fig. 32. Displacement of locating points in the finger electrode area and its correction

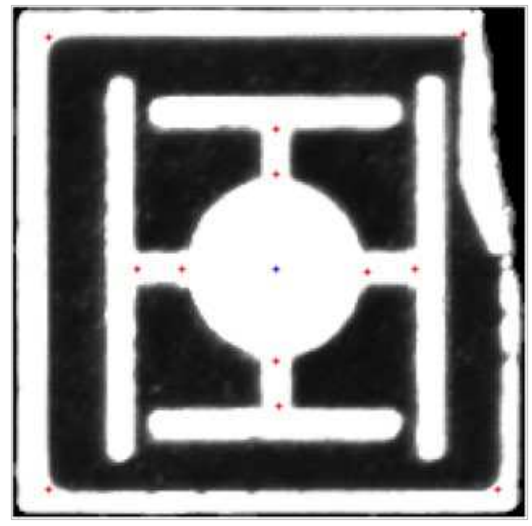

(a) Displacement of locating points in the light-emitting area

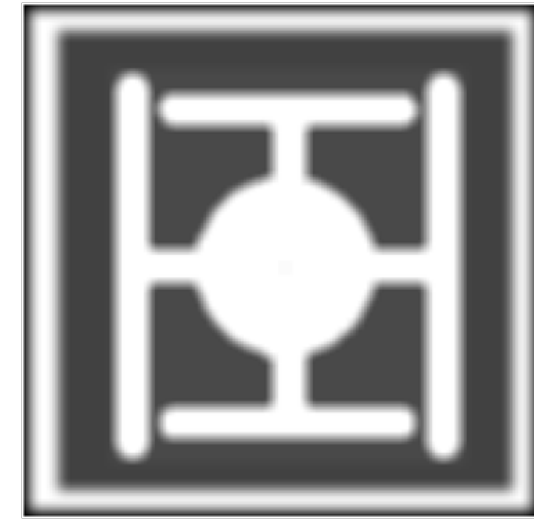

(b) Template with displacement corrected

Fig. 33. Displacement of locating points in the light-emitting area and its correction

\subsection{Detection rate of adaptive template comparison}

The defective areas in the 304 fast-screened LED chips were calculated with the adaptive template comparison system, according to the LED chip detection 
specifications. The defect threshold was 130 pixels in the electrode area, 50 pixels in the light-emitting area, and 180 pixels in the mesa area. The detection rate was analyzed based on the number of statistical defects and the number of actual defects. As a result, 223 chips were correctly judged as non-defective, 76 chips were correctly judged as defective, two chips were overkilled, and three chips were leaked, as shown in Table 6.

Table 6 Misjudgment of adaptive template comparison

\begin{tabular}{|c|c|c|c|c|}
\hline Sample & $\begin{array}{l}\text { Electrode } \\
\text { area }\end{array}$ & $\begin{array}{c}\text { Light- } \\
\text { emitting } \\
\text { area }\end{array}$ & Mesa area & Judgment results \\
\hline $\begin{array}{c}\text { Defect } \\
\text { specification }\end{array}$ & 130 & 50 & 180 & \\
\hline 1 & 0 & 28 & 1 & $\begin{array}{c}\text { Leakage in the light- } \\
\text { emitting area }\end{array}$ \\
\hline 2 & 58 & 12 & 1 & $\begin{array}{l}\text { Leakage in the } \\
\text { electrode area }\end{array}$ \\
\hline 3 & 0 & 17 & 156 & $\begin{array}{c}\text { Leakage in the mesa } \\
\text { area }\end{array}$ \\
\hline 4 & 0 & 109 & 3020 & $\begin{array}{c}\text { Overkill in the mesa } \\
\text { area }\end{array}$ \\
\hline 5 & 0 & 135 & 3458 & $\begin{array}{c}\text { Overkill in the mesa } \\
\text { area }\end{array}$ \\
\hline
\end{tabular}

(Unit: pixels)

As shown in Table 7, the adaptive template comparison method had a correct judgment rate of $99.11 \%$ for non-defective samples and $96.12 \%$ for defective samples. The overall detection rate of the adaptive template comparison method was $98.36 \%$. The system judgment results in the ROC curve of Fig. 34 are also close to the upper left corner, which indicates that the system had a high accuracy 


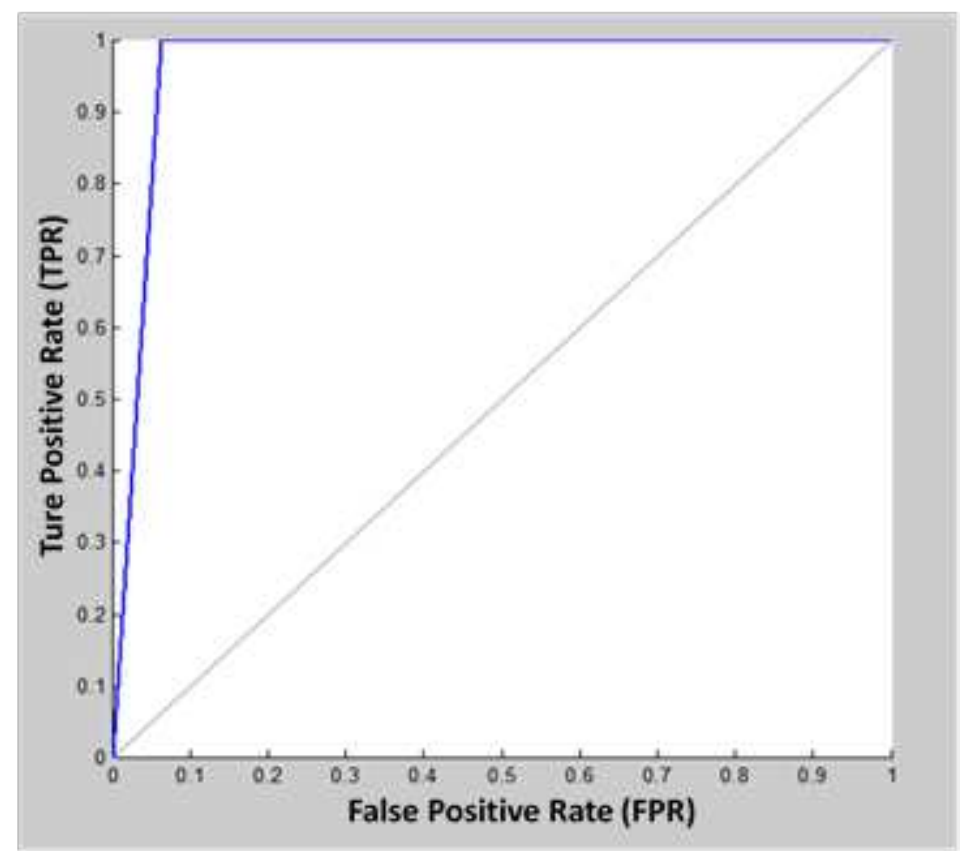

Fig. 34 ROC

Table 7 Confusion matrix of the adaptive template system

\begin{tabular}{|c|c|c|}
\hline & \multicolumn{2}{|c|}{ Actual results } \\
\hline \multirow{2}{*}{ System results } & 223 & 3 \\
\cline { 2 - 3 } & 2 & 76 \\
\hline Total & 225 & 79 \\
\hline
\end{tabular}

The ROC parameters are as follows:

$T P R: \frac{223}{(223+2)}=0.9911$

FPR: $\frac{3}{(3+76)}=0.03801$

$A C C: \frac{(223+76)}{304}=0.9836$ 
During image subtraction, the chips whose defective area was close to the screening threshold or whose defect contrast was low were prone to leakage. Figs. 35 and 36 show the leakage of the defective area with low contrast. In Fig. 37, the defective area of the mesa area is small, but has penetrated the light-emitting area. According to the defect specifications, it was judged as a defective chip, which caused the leakage.

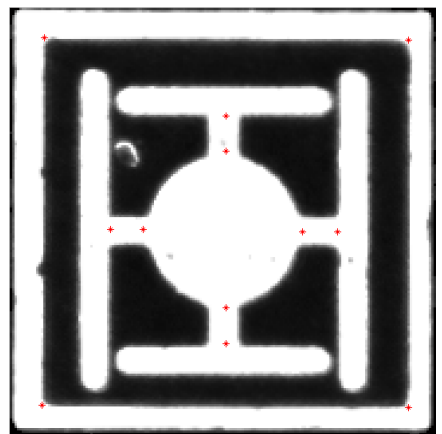

(a) Defects in the lightemitting area

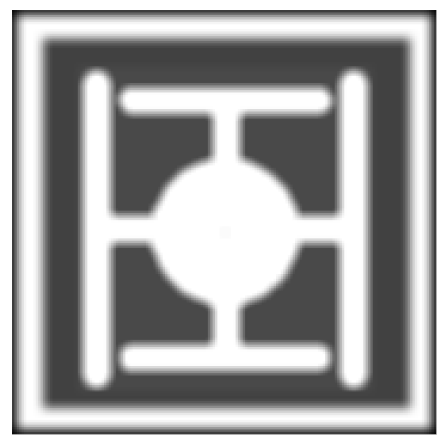

(b) Adaptive template

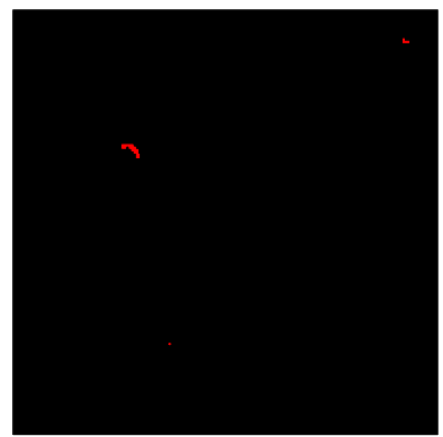

(c) Detection result of the light-emitting area

Fig. 35. Leakage 1 of the adaptive template

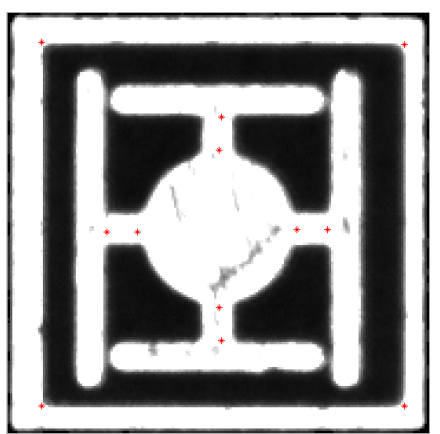

(a) Defects in the light electrode area

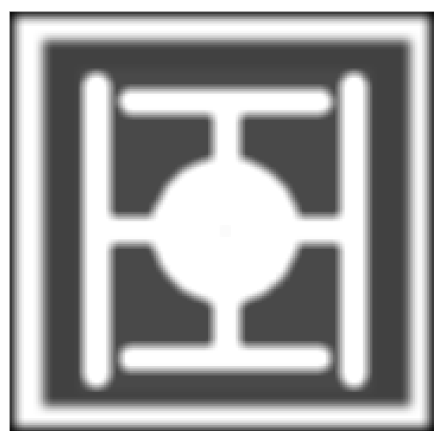

(b) Adaptive template

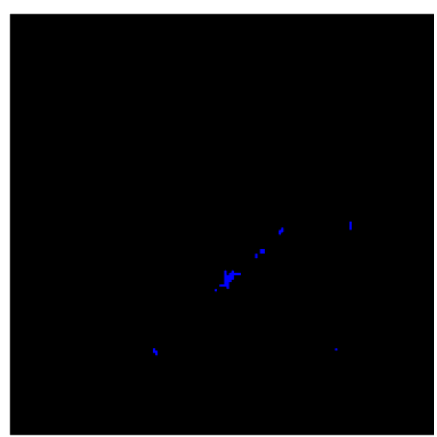

(c) Detection result of the light electrode area

Fig. 36. Leakage 2 of the adaptive template

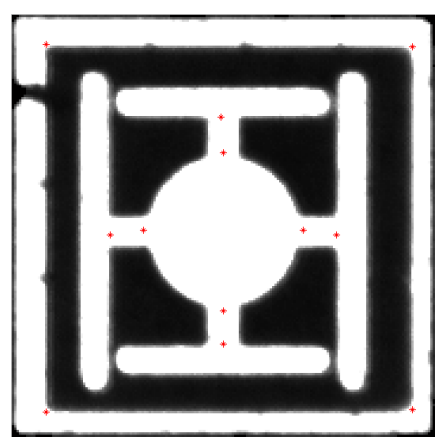

(a) Defects in the mesa

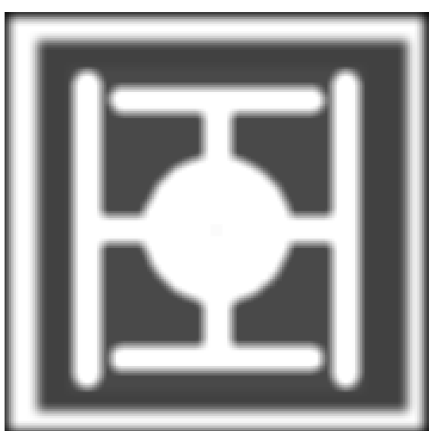

(b) Adaptive template

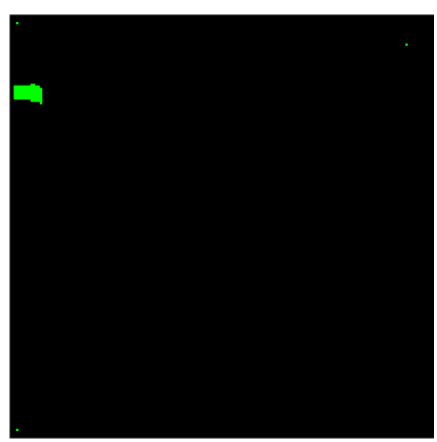

(c) Detection result of the 
Fig. 37. Leakage 3 of the adaptive template

The overkill phenomenon occurred during adaptive template comparison because the positioning was based on the locating points. As the light-emitting area was too close to the chip boundary, two of the four locating points in the light-emitting area were poorly located at the same time. In the actual detection, two chips were subjected to overkill, as shown in Figs. 38 and 39.

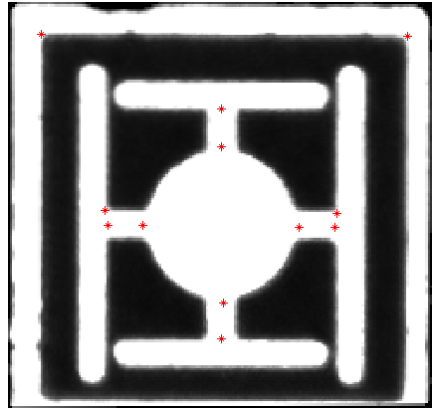

(a) Chip image

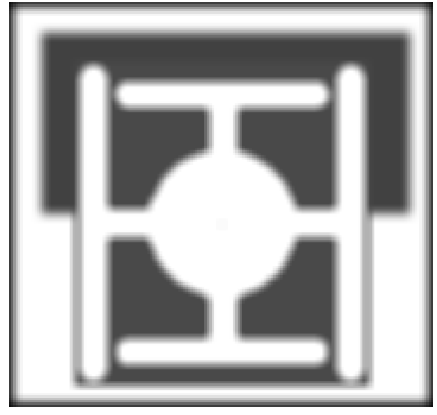

(b) Adaptive template

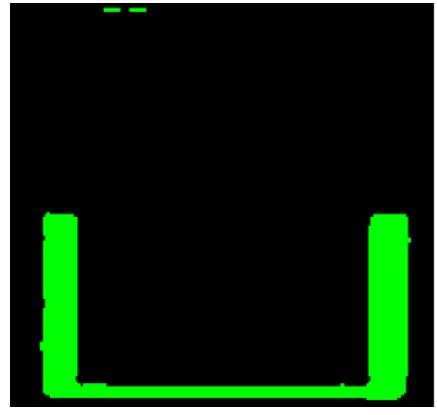

(c) Detection result of the mesa area

Fig. 38. Overkill 1 of the adaptive template

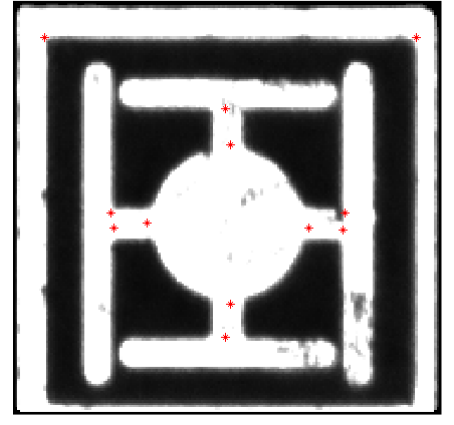

(a) Chip image

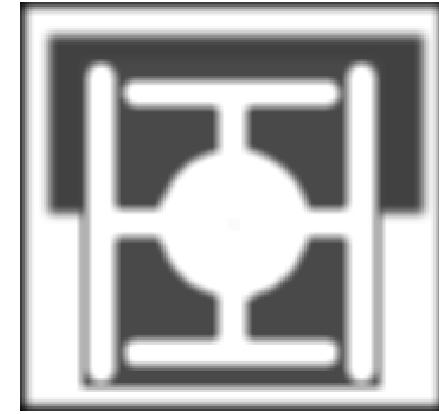

(b) Adaptive template

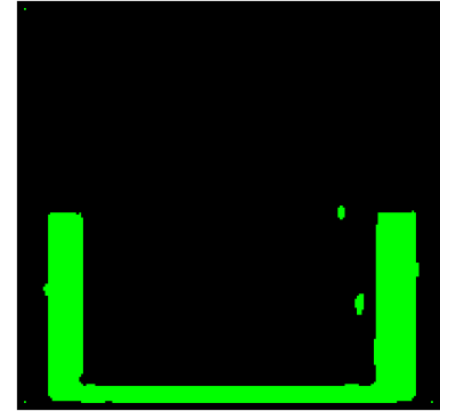

(c) Detection result of the mesa area

Fig. 39. Overkill 2 of the adaptive template

\subsection{Detection and verification of the template comparison method}

Industrial automation detectors often adopt standard template comparison algorithms whose comparison speeds meet the production capacity requirements of the production lines . Regarding the chip samples, there was a huge process error between 
the lithographic patterns of the different mask layers. During detection, the detection parameters must be relaxed to conform to detection standards. The standard template comparison method and the proposed adaptive template comparison method were used to compare and verify the results, as well as to explain the pros and cons of the method.

\subsubsection{Detection rate of the template comparison method}

The template comparison system was used to detect the 304 fast-screened chips. The defects were judged based on the defective areas of the chips, and the number of defective and non-defective chips was obtained to analyze the detection rate. The detection results included the leakage of 32 chips and the overkill of 21 chips. Then, the ROC confusion matrix was used to explore the impact of the template comparison method on the system detection rate and obtain the verification indicators, as shown in Table 8 and Eqs. (21)-(23).

Table 8 Confusion matrix for template comparison

\begin{tabular}{|c|c|c|}
\hline & \multicolumn{2}{|c|}{ Actual results } \\
\hline System results & 204 & 32 \\
\cline { 2 - 3 } & 21 & 47 \\
\hline Total & 225 & 79 \\
\hline
\end{tabular}

The ROC parameters are as follows:

$T P R=\frac{204}{(204+21)}=0.9067$

$F P R=\frac{32}{(32+47)}=0.4051$

$A C C=\frac{(204+47)}{304}=0.8257$ 
The results of the template comparison method show that the correct judgment rate of the non-defective samples was $90.67 \%$, the correct judgment rate of the defective samples was only $59.49 \%$, and the overall detection rate of the template comparison method was $82.57 \%$.

\subsubsection{Detection rate of the adaptive template}

The extracted LED chip sub-images contained a total of 364 chips, including 255 non-defective chips and 139 defective chips. Specifically, 359 chips were correctly judged, three chips were leaked, and two chips were overkilled. From the confusion matrix and related verification indicators of the ROC, the correct judgment rate of the non-defective samples and defective samples and the overall detection rate are deduced. The related equations are shown in Table 9 and Eqs. (24) to (26).

Table 9 Confusion matrix for the adaptive template

\begin{tabular}{|c|c|c|}
\hline & \multicolumn{2}{|c|}{ Actual results } \\
\hline System results & 223 & 3 \\
\cline { 2 - 3 } & 2 & 136 \\
\hline Total & 225 & 139 \\
\hline
\end{tabular}

The ROC parameters are as follows:

$T P R: \frac{223}{(223+3)}=0.9911$

FPR: $\frac{3}{(3+136)}=0.02158$

$A C C: \frac{(223+136)}{(225+139)}=0.9863$

As shown in Table 9, when the fast screening was combined with the adaptive template 
comparison method, the correct judgment rate of the non-defective samples was $99.11 \%$, the correct judgment rate of the defective samples was $97.84 \%$, and the overall detection rate of the template comparison method was $98.63 \%$. The results indicate that the overall detection system in this study had a high detection accuracy.

Comparing the template comparison and the adaptive template methods, we obtain the following table. Establishment of a standard template is the average time from establishing the features of one single chip to extracting the defects, and the detection time is the average detection time for one single chip.

Table 10 Overall index comparison

\begin{tabular}{|c|c|c|c|}
\hline & $\begin{array}{c}\text { Establishment of } \\
\text { standard template }\end{array}$ & Detection time & $\begin{array}{c}\text { Overall detection } \\
\text { accuracy }\end{array}$ \\
\hline $\begin{array}{c}\text { Template } \\
\text { comparison } \\
\text { method }\end{array}$ & $0.33 \mathrm{~s}$ & $4.6 \mathrm{~s}$ & $82.57 \%$ \\
\hline $\begin{array}{c}\text { Adaptive } \\
\text { template }\end{array}$ & $0.25 \mathrm{~s}$ & $3.2 \mathrm{~s}$ & $98.63 \%$ \\
\hline $\begin{array}{c}\text { Efficiency } \\
\text { improvement }\end{array}$ & $0.08 \mathrm{~s}(24.24 \%)$ & $1.4 \mathrm{~s}(30.43 \%)$ & $15.79 \%$ \\
\hline
\end{tabular}

\section{Results and discussions}

Under the same experimental conditions as the adaptive template method, the fast template comparison method was used to detect the chip defects after fast screening and obtain the system detection rate. The displacement of the lithographic patterns between different layers produced a poor fitting of the standard template, as shown in Fig. 40. As a result, the system judged non-defective chips as defective, which led to the overkill phenomenon and greatly increased the number of chip misjudgment. To reduce this phenomenon, the comparison parameters were relaxed to simulate parameter relaxation in the actual detector environment. In the parameter relaxation step, the boundaries of the standard template were processed by a Gaussian blur function and the threshold of defective areas was relaxed to reduce the misjudgment caused by the displacement and scale changes between lithographic patterns. In addition, the defect threshold was adjusted to 150 pixels for the electrode area, 85 pixels for the light-emitting area, and 200 pixels for the mesa area to simulate the case in actual production where the detection parameters will be relaxed to reduce the number of chip 
misjudgment.

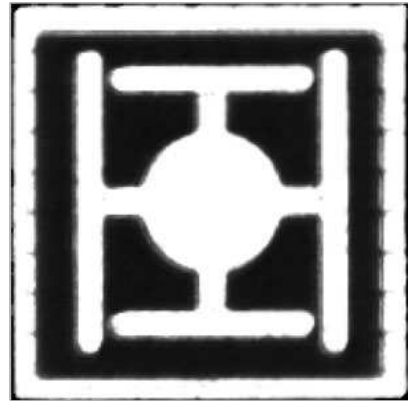

(a) Non-defective chips

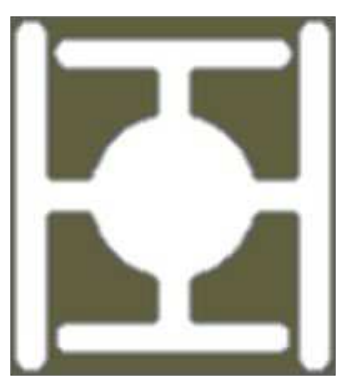

(b) Standard comparison template

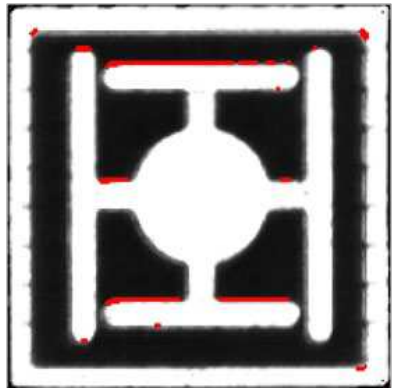

(c) Defect misjudgment

Fig.40. Poor fitting of the template comparison method

The contributions of this study are as follows:

(1) The LED chip images were fast-screened according to the grayscale entropy index and the correlation coefficient index. The severely defective LED chips were removed in advance to increase the locating stability of the adaptive template. It only took $0.052 \mathrm{~s}$ to fast screen one single chip.

(2) SIFT and Harris-Laplace scale space was used to search for and compare the feature points of fast-screened LED chip lithographic patterns. The locating method was innovatively improved to locate feature points by the feature template method for accelerated processes. According to the information of the feature area location points, the standard template of the LED chips was fitted to obtain an adaptive template that matched the chips. It only took $0.14 \mathrm{~s}$ from locating to fitting after the feature area template was improved.

(3) The detection accuracy of the traditional fast template comparison method is only $82.57 \%$ due to the poor fitting between the standard template and the actual chip patterns. In this study, the adjusted adaptive template method had a detection accuracy of $98.36 \%$, which is $15.79 \%$ higher than that of the fast template comparison method. Therefore, the method proposed effectively improved the comparison accuracy. In terms of time performance, it only took $0.25 \mathrm{~s}$ to establish the adaptive template for one single chip. The average detection time of each chip was reduced by $1.4 \mathrm{~s}$, and the efficiency was increased by $30.43 \%$; these values meet the industrial high-speed detection requirements. 


\section{Conclusion}

This study improved the poor fitting between the template and the chip patterns in industrial automated detector algorithms with machine vision and proposed an adaptive template algorithm. Specifically, the feature points of the LED chip lithographic patterns were fitted, and an adaptive template that conformed to the LED chip lithographic patterns was established to detect the chip defects in the object images. The template comparison method and detection rate verification were introduced, which highlighted that the method proposed could improve product detection accuracy. The research samples used in this study were wafer images extracted by an actual industrial detector. The adaptive template can overcome poor fitting by using standard templates and effectively improve the accuracy of the automated optical detectors as well as enhance the industrial production efficiency.

Authors' contributions: Conceptualization, Chung-Feng Jeffrey Kuo; methodology, Cheng-Yu Hung and Wei-Han Weng; validation, Chen-Yang Tsa and Cheng-Yu Hung; formal analysis, Chung-Feng Jeffrey Kuo and Chen-Yang Tsa; data interpretation, WeiHan Weng and Cheng-Yu Hung; writing — original draft preparation, Cheng-Yu Hung; writing - review and editing, Chung-Feng Jeffrey Kuo; visualization, Chen-Yang Tsai; funding acquisition, Chung-Feng Jeffrey Kuo.

All authors have read and agreed to the published version of the manuscript.

Funding: The research was supported by the Ministry of Science and Technology of the Republic of China under Grant No. 109-2221-E-011 -149.

Data availability All data sets generated in this study are available from the corresponding author upon reasonable request.

Compliance with ethical standards

Competing interests The authors declare that they have no competing interests.

Ethical approval Not applicable.

Consent to participate Not applicable.

Consent to publish Not applicable.

\section{References}

[1] Lin H, Li B, Wang X, Shu Y, Niu S (2019) Automated defect inspection of LED chip using deep convolutional neural network. J Intell Manuf 30(6):2525-2534. https://doi.org/10.1007/s10845-018-1415-X

[2] Matsushita Y, Azuno T, Yamashita Y (2012) Fuel reduction in coastal squid jigging boats equipped with various combinations of conventional metal halide lamps and low-energy LED panels. Fish Res 125:14-19. https://doi.org/10.1016/j.fishres.2012.02.004

[3] Kuo CFJ, Hsu CTM, Liu ZX, Wu (2014) HC Automatic inspection system of LED chip using two-stages back-propagation neural network. J Intell Manuf 25(6): 1235-1243. DOI 10.1007/s10845-012-0725-7 
[4] Eckstein HC, Zeitner UD, Leitel R, Stumpf M, Schleicher P, Bräuer A, Tünnermann A (2016) High dynamic grayscale lithography with an LED-based micro-image stepper. In Optical Microlithography XXIX International Society for Optics and Photonics. 9780 97800T. https://doi.org/10.1117/12.2219099.

[5] Choi JW, MacDonald E, Wicker R (2010) Multi-material microstereolithography. Int J Adv Manuf Technol 49(5-8):543-551. DOI 10.1007/s00170-009-2434-8

[6] Peng X, Bennamoun M, Ma Q, Lei Y, Zhang Q, Chen W (2010) Drift-correcting template update strategy for precision feature point tracking. Image Vision Comput 28(8):1280-1292. https://doi.org/10.1016/j.imavis.2010.01.007

[7] Ravishankar (1996) AR Future directions in industrial machine vision: a case study of semiconductor manufacturing applications. Image Vision Comput 14(1): 3-19. https://doi.org/10.1016/0262-8856(95)01035-1

[8] Xiao J, Wei H (2014) Scale-invariant contour segment context in object detection. $\begin{array}{lll}\text { Image Vision } & \text { Comput. } & \text { 32(12):1055-1066 }\end{array}$ https://doi.org/10.1016/j.imavis.2014.08.013

[9] Xie J, Pun CM, Pan Z, Gao H, Wang B (2019) Automatic medical image registration based on an integrated method combining feature and area information. Neural Process Lett 49(1):263-284. https://doi.org/10.1007/s11063-018-9808-6

[10] Wu WY, Hung CW, Yu WB (2013) The development of automated solder bump inspection using machine vision techniques. Int J Adv Manuf Technol 69(14):509-523. https://doi.org/10.1007/s00170-013-4994-X

[11] Kuo CH, Yang FC, Wing JJ, Yang CK (2006) Construction of 3D solder paste surfaces using multi-projection images. Int J Adv Manuf Technol 31(5-6):509-519.

[12] Shi P, Qi Q, Qin Y, Scott PJ, Jiang X (2020) A novel learning-based feature recognition method using multiple sectional view representation. J Intell Manuf 31:1291-1309. https://doi.org/10.1007/s10845-020-01533-w

[13] Fröhlich HB, Grozmani N, Wolfschlaeger D, Goncalves AA, Schmitt RH (2020). Construction of small sets of reference images for feature descriptors fitting and their use in the multiclassification of parts in industry. Int J Adv Manuf Technol 108:105-116. https://doi.org/10.1007/s00170-020-05253-6

[14] Qin A, Guo L, You Z, Gao H, Wu X, Xiang S (2020) Research on automatic monitoring method of face milling cutter wear based on dynamic image sequence. Int J Adv Manuf Technol 110(11):3365-3376. https://doi.org/10.1007/s00170020-05955-X

[15] Yoo JC, Han TH (2009) Fast normalized cross-correlation. Circuits Syst Signal Process 28(6):819. https://doi.org/10.1007/s00034-009-9130-7

[16] Li H, Lee WS, Wang K (2016) Immature green citrus fruit detection and counting based on fast normalized cross correlation (FNCC) using natural outdoor colour images. Precis Agric 17(6):678-697. https://doi.org/10.1007/s11119-016-9443-z

[17] Kuo CFJ, Tsai CH, Wang WR, Wu HC (2019) Automatic marking point positioning of printed circuit boards based on template matching technique. J Intell Manuf 30(2):671-685. https://doi.org/10.1007/s10845-016-1274-2

[18] Stefano Di L, Marchionni M, Mattoccia S (2004) A fast area-based stereo matching algorithm. Image Vision Comput 22(12):983-1005. https://doi.org/10.1016/j.imavis.2004.03.009

[19] Wang Z, Gong S, Li D, Lu H (2017) Error analysis and improved calibration algorithm for LED chip localization system based on visual feedback. Int J Adv Manuf Technol 92(9-12):3197-3206. https://doi.org/10.1007/s00170-017-0390-2

[20] Perng DB, Liu HW, Chang CC (2011) Automated SMD LED inspection using machine vision. Int J Adv Manuf Technol 57(9-12):1065-1077. 
https://doi.org/10.1007/s00170-011-3338-y

[21] Wang L, Chen B, Xu P, Ren H, Fang X, Wan S (2020) Geometry consistency aware confidence evaluation for feature matching. Image Vision Comput 103984. https://doi.org/10.1016/j.imavis.2020.103984

[22] Wang XY, Hou LM, Wu J (2008) A feature-based robust digital image watermarking against geometric attacks. Image Vision Comput 26(7):980-989. https://doi.org/10.1016/j.imavis.2007.10.014

[23] Harris CG, Stephens M (1988, August) A combined corner and edge detector. In Alvey vision conference 15(50):10-5244. doi:10.5244/C.2.23

[24] Leutenegger S, Lynen S, Bosse M, Siegwart R, Furgale P (2015) Keyframe-based visual-inertial odometry using nonlinear optimization. Int J Robot Res 34(3):314334. https://doi.org/10.1177/0278364914554813

[25] Lowry S, Sünderhauf N, Newman P, Leonard JJ, Cox D, Corke P, Milford MJ (2015) Visual place recognition: A survey. Int $\mathbf{J}$ Robot Res 32(1):1-19. 10.1109/TRO.2015.2496823

[26] Smith SM, Brady JM, (1997) SUSAN — a new approach to low level image $\begin{array}{lllll}\text { processing. Int } & \mathrm{J} \text { Comput }\end{array}$ https://oi.org/10.1023/A:1007963824710

[27] Chen LC, Papandreou G, Kokkinos I, Murphy K, Yuille AL (2017) Deeplab: Semantic image segmentation with deep convolutional nets, atrous convolution, and fully connected crfs. IEEE Trans Pattern Anal 40(4):834-848. 10.1109/TPAMI.2017.2699184

[28] Zhong F, He S, Li B (2017) Blob analyzation-based template matching algorithm for LED chip localization. Int $J$ Adv Manuf Technol 93(1-4):55-63. https://doi.org/10.1007/s00170-015-7638-5

[29] Yan Z, Shi B, Sun L, Xiao J (2020) Surface defect detection of aluminum alloy welds with 3D depth image and 2D gray image. Int J Adv Manuf Technol 110(3): 741-752. https://doi.org/10.1007/s00170-020-05882-x

[30] Lowe DG (1999, September) Object recognition from local scale-invariant features. In Proceedings of the seventh IEEE international conference on computer vision 2:1150-1157. 10.1109/ICCV.1999.790410

[31] Schmidhuber J (2015) Deep learning in neural networks: An overview. Neural Netw 61:85-117. https://doi.org/10.1016/j.neunet.2014.09.003

[32] Sullivan GD, Baker KD, Anderson JADW (1985) Use of multiple difference-ofGaussian filters to verify geometric models. Image Vision Comput. 3(4):192-197. https://doi.org/10.1016/0262-8856(85)90007-1

[33] Se S, Lowe DG, Little JJ (2005) Vision-based global localization and mapping for mobile robots. IEEE Trans Robot 21(3):364-375. 10.1109/TRO.2004.839228

[34] Fuentes-Pacheco J, Ruiz-Ascencio J, Rendón-Mancha JM (2015) Visual simultaneous localization and mapping: a survey. Artif Intell Rev 43(1):55-81. DOI 10.1007/s10462-012-9365-8

[35] Bonin-Font F, Ortiz A, Oliver G (2008) Visual navigation for mobile robots: A survey. J Intell Robot Syst 53(3):263. https://doi.org/10.1007/s10846-008-9235-4

[36] Bresson G, Alsayed Z, Yu L, Glaser S (2017) Simultaneous localization and mapping: A survey of current trends in autonomous driving. IEEE Trans Intell Veh 2(3):194-220. 10.1109/TIV.2017.2749181

[37] Mikolajczyk K, Schmid C (2001, July) Indexing based on scale invariant interest points. In Proceedings Eighth IEEE International Conference on Computer Vision ICCV 1:525-531. 10.1109/ICCV.2001.937561

[38] Dorkó G, Schmid C (2003, October) Selection of Scale-Invariant Parts for Object 
Class Recognition. ICCV 3:634. 10.1109/ICCV.2003.1238407

[39] Mikolajczyk K, Schmid C (2004) Scale \& affine invariant interest point detectors. Int J Comput Vis 60(1):63-86. https://doi.org/10.1023/B:VISI.0000027790.02288.f2

[40] Gordo A, Almazán J, Revaud J, Larlus D (2016, October) Deep image retrieval: Learning global representations for image search. In European conference on computer vision 241-257. https://doi.org/10.1007/978-3-319-46466-4_15

[41] Korytkowski M, Rutkowski L, Scherer R (2016) Fast image classification by boosting fuzzy classifiers. Inf Sci 327:175-182. https://doi.org/10.1016/j.ins.2015.08.030

[42] Zhang J, Chen Q, Sun Q, Sun H, Xia D (2011) A highly repeatable feature detector: improved Harris-Laplace. Multimed Tools Appl 52(1):175-186. https://doi.org/10.1007/s11042-010-0471-9

[43] Fawcett T (2006) An introduction to ROC analysis. Pattern Recognit Lett 27(8): 861-874. https://doi.org/10.1016/j.patrec.2005.10.010

[44] Chen D, P Wang, Yue L, Zhang Y, Jia T (2020) Anomaly detection in surveillance video based on bidirectional prediction. Image Vision Comut 103915. https://doi.org/10.1016/j.imavis.2020.103915

[45] Mason SE, Nicolay CR, Darzi (2015) A The use of Lean and Six Sigma methodologies in surgery: A systematic review. The Surgeon 13(2):91-100. https://doi.org/10.1016/j.surge.2014.08.002

[46] Kwak YH, Anbari FT (2006) Benefits, obstacles, and future of six sigma approach. Technovation 26(5-6):708-715. https://doi.org/10.1016/j.technovation.2004.10.003 